\title{
Pseudo almost periodic functions and pseudo almost periodic solutions to dynamic equations on time scales
}

Yongkun Li* and Chao Wang

* Correspondence: yklie@ynu.edu. $\mathrm{cn}$

Department of Mathematics, Yunnan University Kunming, Yunnan 650091, People's Republic of China

\begin{abstract}
In this paper, we first introduce a concept of the mean-value of uniformly almost periodic functions on time scales and give some of its basic properties. Then, we propose a concept of pseudo almost periodic functions on time scales and study some basic properties of pseudo almost periodic functions on time scales. Finally, we establish some results about the existence of pseudo almost periodic solutions to dynamic equations on time scales.
\end{abstract}

Keywords: dynamic equations on time scales, pseudo almost periodic functions, exponential dichotomy, pseudo almost periodic solutions

\section{Introduction}

The theory of dynamic equations on time scales has been developed over the last several decades, it has been created in order to unify the study of differential and difference equations. Many papers have been published on the theory of dynamic equations on time scales [1-14]. In addition, the existence of almost periodic, asymptotically almost periodic, pseudo-almost periodic solutions is among the most attractive topics in the qualitative theory of differential equations and difference equations due to their applications, especially in biology, economics and physics [15-34]. Recently, in [14,35], the almost periodic functions and the uniformly almost periodic functions on time scales were presented and investigated, as applications, the existence of almost periodic solutions to a class of functional differential equations and neural networks were studied effectively (see $[13,14,35]$ ). However, there is no concept of pseudo-almost periodic functions on time scales so that it is impossible for us to study pseudo almost periodic solutions for dynamic equations on time scales.

Motivated by the above, our main purpose of this paper is firstly to introduce a concept of mean-value of uniformly almost periodic functions and give some useful and important properties of it. Then we propose a concept of pseudo almost periodic functions which is a new generalization of uniformly almost periodic functions on time scales and present some relative results. Finally, we establish some results about the existence and uniqueness of pseudo almost periodic solutions to dynamic equations on time scales.

The organization of this paper is as follows: In Section 2, we introduce some notations, definitions and state some preliminary results needed in the later sections. In

(C) $2012 \mathrm{Li}$ and Wang; licensee Springer. This is an Open Access article distributed under the terms of the Creative Commons Attribution License (http://creativecommons.org/licenses/by/2.0), which permits unrestricted use, distribution, and reproduction in any medium, provided the original work is properly cited. 
Section 3, we introduce a concept of mean-value of uniformly almost periodic functions and establish some useful and important results. In Section 4, we propose a concept of pseudo almost periodic functions on time scales and present some relative results. In Section 5, we establish some results about the existence and uniqueness of pseudo almost periodic solutions to dynamic equations on time scales. As applications of our results, in Section 6, we study the existence of pseudo almost periodic solutions to quasi-linear dynamic equations on time scales.

\section{Preliminaries}

Let $\mathbb{T}$ be a nonempty closed subset (time scale) of $\mathbb{R}$. The forward and backward jump operators $\sigma, \rho: \mathbb{T} \rightarrow \mathbb{T}$ and the graininess $\mu: \mathbb{T} \rightarrow \mathbb{R}^{+}$are defined, respectively, by

$$
\sigma(t)=\inf \{s \in \mathbb{T}: s>t\}, \quad \rho(t)=\sup \{s \in \mathbb{T}: s<t\}, \quad \mu(t)=\sigma(t)-t .
$$

A point $t \in \mathbb{T}$ is called left-dense if $t>\inf \mathbb{T}$ and $\rho(t)=t$, left-scattered if $\rho(t)<t$, right-dense if $t<\sup \mathbb{T}$ and $\sigma(t)=t$, and right-scattered if $\sigma(t)>t$. If $\mathbb{T}$ has a left-scattered maximum $m$, then $\mathbb{T}^{k}=\mathbb{T} \backslash\{m\}$; otherwise $\mathbb{T}^{k}=\mathbb{T}$. If $\mathbb{T}$ has a right-scattered minimum $m$, then $\mathbb{T}_{k}=\mathbb{T} \backslash\{m\}$; otherwise $\mathbb{T}_{k}=\mathbb{T}$.

A function $f: \mathbb{T} \rightarrow \mathbb{R}$ is right-dense continuous provided that it is continuous at right-dense point in $\mathbb{T}$ and its left-side limits exist at left-dense points in $\mathbb{T}$. If $f$ is continuous at each right-dense point and each left-dense point, then $f$ is said to be a continuous function on $\mathbb{T}$.

For $y: \mathbb{T} \rightarrow \mathbb{R}$ and $t \in \mathbb{T}^{k}$, we define the delta derivative of $y(t), y^{\Delta}(t)$, to be the number (if it exists) with the property that for a given $\varepsilon>0$, there exists a neighborhood $U$ of $t$ such that

$$
\left|[\gamma(\sigma(t))-\gamma(s)]-\gamma^{\Delta}(t)[\sigma(t)-s]\right|<\varepsilon|\sigma(t)-s|
$$

for all $s \in U$.

Let $y$ be right-dense continuous, if $Y^{\Delta}(t)=y(t)$, then we define the delta integral by

$$
\int_{a}^{t} \gamma(s) \Delta s=Y(t)-Y(a)
$$

A function $p: \mathbb{T} \rightarrow \mathbb{R}$ is called regressive provided $1+\mu(t) p(t) \neq 0$ for all $t \in \mathbb{T}^{k}$. The set of all regressive and rd-continuous functions $p: \mathbb{T} \rightarrow \mathbb{R}$ will be denoted by $\mathcal{R}=\mathcal{R}(\mathbb{T})=\mathcal{R}(\mathbb{T}, \mathbb{R}) . \quad$ We $\quad$ define the $\mathcal{R}^{+}=\mathcal{R}^{+}(\mathbb{T}, \mathbb{R})=\{p \in \mathcal{R}: 1+\mu(t) p(t)>0, \forall t \in \mathbb{T}\}$.

A $n \times n$-matrix-valued function $A$ on a time scale $\mathbb{T}$ is called regressive provided $I+$ $\mu(t) A(t)$ is invertible for all $t \in \mathbb{T}$, and the class of all such regressive and rd-continuous functions is denoted, similar to the above scalar case, by $\mathcal{R}=\mathbb{R}(\mathbb{T})=\mathcal{R}\left(\mathbb{T}, \mathbb{R}^{n \times n}\right)$.

If $r$ is a regressive function, then the generalized exponential function $e_{r}$ is defined by

$$
e_{r}(t, s)=\exp \left\{\int_{s}^{t} \xi_{\mu(\tau)}(r(\tau)) \Delta \tau\right\}
$$

for all $\mathrm{s}, t \in \mathbb{T}$, with the cylinder transformation

$$
\xi_{h}(z)=\left\{\begin{array}{l}
\frac{\log (1+\mathrm{hz})}{h}, \text { if } h \neq 0, \\
z, \quad \text { if } h=0 .
\end{array}\right.
$$


Definition 2.1 ([1,3]). Let $p, q: \mathbb{T} \rightarrow \mathbb{R}$ be two regressive functions, define

$$
p \oplus q=p+q+\mu p q, \quad \ominus p=-\frac{p}{1+\mu p}, \quad p \ominus q=p \oplus(\ominus q) .
$$

Lemma 2.1 ([1,3]). Assume that $p, q: \mathbb{T} \rightarrow \mathbb{R}$ are two regressive functions, then

(i) $e_{0}(t, s) \equiv 1$ and $e_{p}(t, t) \equiv 1$;

(ii) $e_{p}(\sigma(t), s)=(1+\mu(t) p(t)) e_{p}(t, s)$;

(iii) $e_{p}(t, s)=\frac{1}{e_{p}(s, t)}=e_{\ominus p}(s, t) ; e_{p}(t, s) e_{p}(s, r)=e_{p}(t, r)$;

(iv) $\left(e_{\ominus p}(t, s)\right)^{\Delta}=(\ominus p)(t) e_{\ominus p}(t, s)$;

(v) If $a, b, c \in \mathbb{T}$, then $\int_{a}^{b} p(t) e_{p}(c, \sigma(t)) \Delta t=e_{p}(c, a)-e_{p}(c, b)$.

Definition 2.2 ([36]). For every $x, y \in \mathbb{R},[x, y)=\{t \in \mathbb{R}: x \leq t<y\}$, define a countably additive measure $m_{1}$ on the set

$$
\mathcal{F}_{1}=\{[\tilde{a}, \tilde{b}) \cap \mathbb{T}: \tilde{a}, \tilde{b} \in \mathbb{T}, \tilde{a} \leq \tilde{b}\},
$$

that assigns to each interval $[\tilde{a}, \tilde{b}) \cap \mathbb{T}$ its length, that is,

$$
m_{1}([\tilde{a}, \tilde{b}))=\tilde{b}-\tilde{a} .
$$

The interval $[\tilde{a}, a)$ is understood as the empty set. Using $m_{1}$, they generate the outer measure $m_{1}^{*}$ on $\mathcal{P}(\mathbb{T})$, defined for each $E \in \mathcal{P}(\mathbb{T})$ as

$$
m_{1}^{*}(E)=\left\{\begin{array}{lr}
\inf _{\tilde{\mathcal{R}}}\left\{\sum_{i \in I_{\tilde{\mathcal{R}}}}\left(\tilde{b}_{i}-\tilde{a}_{i}\right)\right\} \in \mathbb{R}^{+}, b \notin E \\
+\infty, & b \in E
\end{array}\right.
$$

with

$$
\tilde{\mathcal{R}}=\left\{\left\{\left[\tilde{a}_{i}, \tilde{b}_{i}\right) \cap \mathbb{T} \in \mathcal{F}_{1}\right\}_{i \in I_{\tilde{\mathcal{R}}}}: I_{\tilde{\mathcal{R}}} \subset N, E \subset \cup_{i \in I_{\tilde{\mathcal{R}}}}\left(\left[a_{i}, b_{i}\right) \cap \mathbb{T}\right)\right\} .
$$

$A$ set $A \subset \mathbb{T}$ is said to be $\Delta$-measurable if the following equality:

$$
m_{1}^{*}(E)=m_{1}^{*}(E \cap A)+m_{1}^{*}(E \cap(\mathbb{T} \backslash A))
$$

holds true for all subset $E$ of $\mathbb{T}$. Define the family

$$
\mathcal{M}\left(m_{1}^{*}\right)=\{A \subset \mathbb{T}: \text { Ais } \Delta-\text { measurable }\},
$$

the Lebesgue $\Delta$-measure, denoted by $\mu_{\Delta}$, is the restriction of $m_{1}^{*}$ to $\mathcal{M}\left(m_{1}^{*}\right)$.

Definition 2.3 ([35]). A time scale Tis called an almost periodic time scale if

$$
\Pi:=\{\tau \in \mathbb{R}: t \pm \tau \in \mathbb{T}, \forall t \in \mathbb{T}\} \neq\{0\} .
$$

Remark 2.1. In the following, we always use $\mathbb{T}$ to denote an almost periodic time scale. Throughout this paper, $\mathbb{E}^{n}$ denotes $\mathbb{R}^{n}$ or $\mathbb{C}^{n}, D$ denotes an open set in $\mathbb{E}^{n}$ or $D=\mathbb{E}^{n}$, $S$ denotes an arbitrary compact subset of $D$.

Definition 2.4 ([35]). Let $\mathbb{T}$ be an almost periodic time scale. A function $f \in C\left(\mathbb{T} \times D, \mathbb{E}^{n}\right)$ is called an almost periodic function in $t \in \mathbb{T}$ uniformly for $x \in D$ if the $\varepsilon$-translation set of $f$

$$
E\{\varepsilon, f, S\}=\{\tau \in \Pi:|f(t+\tau, x)-f(t, x)|<\varepsilon, \text { for all }(t, x) \in \mathbb{T} \times S\}
$$


is a relatively dense set in $\mathbb{T}$ for all $\varepsilon>0$ and for each compact subset $S$ of $D$; that is, for any given $\varepsilon>0$ and each compact subset $S$ of $D$, there exists a constant $l(\varepsilon, S)>0$ such that each interval of length $l(\varepsilon, S)$ contains a $\tau(\varepsilon, S) \in E\{\varepsilon, f, S\}$ such that

$$
|f(t+\tau, x)-f(t, x)|<\varepsilon, \quad \text { for all } t \in \mathbb{T} \times S .
$$

$\tau$ is called the $\varepsilon$-translation number of $f$ and and $l(\varepsilon, S)$ is called the inclusion length of $E\{\varepsilon, f, S\}$.

\section{The mean-value of uniformly almost periodic functions on time scales}

Let $f \in C\left(\mathbb{T} \times D, \mathbb{E}^{n}\right)$ and $f(t, x)$ be almost periodic in $t$ uniformly for $x \in D$, we denote

$$
a(f, \lambda, x):=\lim _{T \rightarrow+\infty} \frac{1}{T} \int_{t_{0}}^{t_{0}+T} f(t, x) e^{-i \lambda t} \Delta t, \quad \text { where } t_{0} \in \mathbb{T}, T \in \Pi,
$$

where $\lambda \in \mathbb{R}, i=\sqrt{-1}$. Obviously, for a fixed $(f, \lambda, x), a(f, \lambda, x) \in \mathbb{E}^{n}$.

Definition 3.1. $a(f(t, 0, x))$ is called mean-value of $f(t, x)$ if

$$
0<a(f, 0, x)=\lim _{T \rightarrow \infty} \frac{1}{T} \int_{t_{0}}^{t_{0}+T} f(t, x) \Delta t<+\infty .
$$

Theorem 3.1. For any $\lambda \in \mathbb{R}, a(f, \lambda, x)$ defined by (3.1) exists uniformly for $x \in S$ and is uniformly continuous on $S$ with respect to $x$, where $S$ is an arbitrary compact subset of $D$.

Proof. For any $t_{1} \in \Pi, t_{1}>0$, we can make a sequence $\left\{t_{i}\right\}_{i \in \mathbb{Z}^{+}} \subset \prod$, where $t_{i}=i t_{1}$. We will prove that the sequence $\left\{\frac{1}{t_{i}} \int_{t_{0}}^{t_{0}+t_{i}} f(t, x) \Delta t\right\}_{i \in \mathbb{Z}^{+}}$converges uniformly with respect to $x \in S$.

For any integers $m, n$ and $x \in S$, taking $t_{m}, t_{n}$, we have

$$
\begin{aligned}
\left|\frac{1}{t_{n}} \int_{t_{0}}^{t_{0}+t_{n}} f(t, x) \Delta t-\frac{1}{t_{m}} \int_{t_{0}}^{t_{0}+t_{m}} f(t, x) \Delta t\right| \\
\leq \frac{1}{t_{m} t_{n}}\left|t_{m} \int_{t_{0}}^{t_{0}+t_{n}} f(t, x) \Delta t-\int_{t_{0}}^{t_{0}+t_{m n}} f(t, x) \Delta t\right| \\
\quad+\frac{1}{t_{m} t_{n}}\left|\int_{t_{0}}^{t_{0}+t_{m n}} f(t, x) \Delta t-t_{n} \int_{t_{0}}^{t_{0}+t_{m}} f(t, x) \Delta t\right| \\
\leq\left|\frac{t_{1}}{t_{m} t_{n}}\right|\left[\sum_{k=1}^{m}\left|\int_{t_{0}}^{t_{0}+t_{n}} f(t, x) \Delta t-\int_{t_{(k-1) n}}^{t_{0}+t_{m n}} f(t, x) \Delta t\right|\right. \\
\left.+\sum_{k=1}^{n}\left|\int_{(k-1) m}^{t_{0}+t_{m m}} f(t, x) \Delta t-\int_{t_{0}}^{t_{0}+t_{m}} f(t, x) \Delta t\right|\right] .
\end{aligned}
$$


Consider the following integral form:

$$
\int_{t_{a}}^{t_{0}+t_{a+s}} f(t, x) \Delta t-\int_{t_{0}}^{t_{0}+t_{s}} f(t, x) \Delta t
$$

where $s=n, a=(k-1) n, k=1,2, \ldots, m$ or $s=m, a=(k-1) m, k=1,2, \ldots, n$. For arbitrary $a$, $s$, we can evaluate (3.3):

For any $\varepsilon>0$, let $l=l\left(\frac{\varepsilon}{4}, S\right)$ be an inclusion length of $E\left(f, \frac{\varepsilon}{4}, S\right)$ and $\tau \in E\left(f, \frac{\varepsilon}{4}, S\right) \cap\left[t_{a}-t_{0}, t_{a}-t_{0}+l\right]$, then, for all $x \in S$, we get ${ }^{* * * *}$

$$
\begin{aligned}
& \left|\int_{t_{a}}^{t_{0}+t_{a+s}} f(t, x) \Delta t-\int_{t_{0}}^{t_{0}+t_{s}} f(t, x) \Delta t\right| \\
= & \left|\int_{t_{0}+\tau}^{t_{0}+\tau+t_{s}}-\int_{t_{0}}^{t_{0}+t_{s}}+\int_{t_{0}+\tau+t_{s}}^{t_{0}+t_{a+s}}+\int_{t_{a}}^{t_{0}+\tau}\right| f(t, x) \Delta t \mid \\
\leq & \int_{t_{0}}^{t_{0}+t_{s}}|f(t+\tau, x)-f(t, x)| \Delta t+\int_{t_{0}+\tau+t_{s}}^{t_{0}+t_{a+s}}|f(t, x)| \Delta t+\int_{t_{a}}^{t_{0}+\tau}|f(t, x)| \Delta t \\
\leq & \frac{\varepsilon t_{s}}{4}+2 l G,
\end{aligned}
$$

where $G=\sup _{(t, x) \in \mathbb{T} \times S}|f(t, x)|$. According to (3.4), we can reduce (3.2) to the following:

$$
\begin{aligned}
\left|\frac{1}{t_{n}} \int_{t_{0}}^{t_{0}+t_{n}} f(t) \Delta t-\frac{1}{t_{m}} \int_{t_{0}}^{t_{0}+t_{m}} f(t) \Delta t\right| & <\frac{t_{1}}{t_{m} t_{n}}\left[m\left(\frac{\varepsilon t_{n}}{4}+2 l G\right)+n\left(\frac{\varepsilon t_{m}}{4}+2 l G\right)\right] \\
& =\frac{\varepsilon}{2}+\frac{2 l G}{t_{1}}\left(\frac{1}{m}+\frac{1}{n}\right) \rightarrow 0, \quad t_{m}, t_{n} \rightarrow+\infty .
\end{aligned}
$$

By the Cauchy convergence criterion, the sequence $\left\{\frac{1}{t_{i}} \int_{t_{0}}^{t_{0}+t_{i}} f(t, x) \Delta t\right\}_{i \in \mathbb{N}}$ converges uniformly with respect to $x \in S$.

For any sufficiently large $0<T \in \Pi$, there exist $0<t_{n} \in \Pi$ such that $0<t_{n}<T \leq t_{n+1}$, so for all $x \in S$, we have

$$
\left|\int_{t_{0}}^{t_{0}+T} f(t, x) \Delta t-\int_{t_{0}}^{t_{0}+t_{n}} f(t, x) \Delta t\right| \leq G\left(T-t_{n}\right) \leq G t_{1} .
$$

Therefore,

$$
\begin{aligned}
\left|\frac{1}{T} \int_{t_{0}}^{t_{0}+T} f(t, x) \Delta t-\frac{1}{t_{n}} \int_{t_{0}}^{t_{0}+t_{n}} f(t, x) \Delta t\right|< & \frac{1}{T}\left|\int_{t_{0}}^{t_{0}+T} f(t, x) \Delta t-\int_{t_{0}}^{t_{0}+t_{n}} f(t, x) \Delta t\right| \\
& +\left(\frac{1}{t_{n}}-\frac{1}{T}\right) \int_{t_{0}}^{t_{0}+t_{n}}|f(t, x)| \Delta t \\
\leq & \frac{G t_{1}}{T}+\left(\frac{1}{t_{n}}-\frac{1}{T}\right) t_{n} G \\
& <\frac{2 G}{n} \rightarrow 0, \quad t_{n} \rightarrow+\infty .
\end{aligned}
$$


Hence,

$$
a(f, 0, x)=\lim _{n \rightarrow+\infty} \frac{1}{t_{n}} \int_{t_{0}}^{t_{0}+t_{n}} f(t, x) \Delta t \text { uniformly for } x \in S .
$$

Besides, for $\frac{1}{T} \int_{t_{0}}^{t_{0}+T} f(t, x) \Delta t$ is continuous with respect to $x \in S$, where $S$ is an arbitrary compact set in $\mathbb{E}^{n}, a(f, 0, x)$ is uniformly continuous on $S$.

It is oblivious that $f(t, x) e^{-i \lambda t}$ is almost periodic in $t$ uniformly for $x \in D$ and $a(f, \lambda, x)$ $=a\left(f(t, x) e^{-i \lambda t}, 0, x\right)$, so it is easy to see that $a(f, \lambda, x)$ exists uniformly for $x \in S$ and is uniformly continuous on $S$ with respect to $x$. This completes the proof. $\square$

Theorem 3.2. Assume that $T \in \Pi$ and $f(t, x) \in C\left(\mathbb{T} \times D, \mathbb{E}^{n}\right)$ is almost periodic in $t$ uniformly for $x \in D$, then

$$
\lim _{T \rightarrow+\infty} \frac{1}{T} \int_{\alpha}^{\alpha+T} f(t, x) e^{-i \lambda t} \Delta t:=m(f(t, x), \lambda, x)
$$

uniformly exists for $\alpha \in \mathbb{T}$ and

$$
m(f(t, x), \lambda, x)=a\left(f(t+\alpha, x) e^{-i \lambda \alpha}, \lambda, x\right) .
$$

Proof. For $m(f, \lambda, x)=m\left(f(t, x) e^{-i \lambda t}, 0, x\right)$, it suffices to show that, for $x \in S, \forall \alpha \in \mathbb{T}$, the following uniformly exists:

$$
m(f, 0, x)=\lim _{T \rightarrow+\infty} \frac{1}{T} \int_{\alpha}^{\alpha+T} f(t, x) \Delta t .
$$

Take $l=l\left(\frac{\varepsilon}{4}, S\right)$ and $\tau \in E\left\{\frac{\varepsilon}{4}, f, S\right\} \cap\left[\alpha-t_{0}, \alpha-t_{0}+l\right], G=\sup _{(t, x) \in \mathbb{T} \times S}|f(t, x)|$, for $x \in$ $S$, we obtain

$$
\begin{aligned}
& \left|\frac{1}{T} \int_{\alpha}^{\alpha+T} f(t, x) \Delta t-\frac{1}{T} \int_{t_{0}}^{t_{0}+T} f(t, x) \Delta t\right| \\
& =\frac{1}{T}\left|\left(\int_{t_{0}+\tau}^{t_{0}+\tau+T}-\int_{t_{0}}^{t_{0}+T}+\int_{t_{0}+\tau+T}^{\alpha+T}+\int_{\alpha}^{t_{0}+\tau}\right) f(t, x) \Delta t\right| \\
& \quad \leq \frac{1}{T}\left(\int_{t_{0}}^{t_{0}+T}|f(t+\tau, x)-f(t, x)| \Delta t+\int_{t_{0}+\tau+T}^{\alpha+T}|f(t, x)| \Delta t+\int_{\alpha}^{t_{0}+\tau}|f(t, x)| \Delta t\right) \\
& \quad \leq \frac{1}{T}\left(\frac{\varepsilon T}{4}+\frac{2 l G}{T}\right)=\frac{\varepsilon}{4}+\frac{2 l G}{T}
\end{aligned}
$$

and

$$
\begin{aligned}
& \left|\frac{1}{n T} \int_{t_{0}}^{t_{0}+n T} f(t, x) \Delta t-\frac{1}{T} \int_{t_{0}}^{t_{0}+T} f(t, x) \Delta t\right| \\
= & \frac{1}{n}\left|\sum_{k=1}^{n} \frac{1}{T}\left[\int_{t_{0}+(k-1) T}^{t_{0}+k T} f(t, x) \Delta t-\int_{t_{0}}^{t_{0}+T} f(t, x) \Delta t\right]\right| \\
\leq & \frac{\varepsilon}{4}+\frac{2 l G}{T} .
\end{aligned}
$$


From (3.7), let $n \rightarrow \infty$, for $x \in S$, we have

$$
\left|a(f, 0, x)-\frac{1}{T} \int_{t_{0}}^{t_{0}+T} f(t, x) \Delta t\right| \leq \frac{\varepsilon}{4}+\frac{2 l G}{T} .
$$

Using trigonometric inequality, according (3.6) and (3.8), we can take $T>\frac{8 l G}{\varepsilon}$ such that

$$
\left|\frac{1}{T} \int_{\alpha}^{\alpha+T} f(t, x) \Delta t-a(f, 0, x)\right| \leq \frac{\varepsilon}{2}+\frac{4 l G}{T}<\varepsilon .
$$

Hence, we can easily obtain that (3.5) uniformly exists for $\alpha \in \mathbb{T}$ and $m(f, 0, x)=a(f$, $0, x)=a(f(t, x), 0, x)$. Furthermore,

$$
\frac{1}{T} \int_{\alpha}^{\alpha+T} f(t, x) \Delta t=\frac{1}{T} \int_{t_{0}}^{t_{0}+T} f(t+\alpha, x) \Delta t
$$

Therefore, $a(f(t+\alpha, x), 0, x)$ uniformly exists for $\alpha \in \mathbb{T}$ and $m(f(t, x), 0, x)=a(f(t+$ $\alpha, x), 0, x)$. It is easy to see that $f(t, x) e^{-i \lambda t}$ is almost periodic in $t$ uniformly for $x \in D$, thus, we have

$$
\begin{aligned}
m(f(t, x), \lambda, x) & =m\left(f(t, x) e^{-i \lambda t}, 0, x\right) \\
& =a\left(f(t+\alpha, x) e^{-i \lambda(t+\alpha)}, 0, x\right) \\
& =a\left(f(t+\alpha, x) e^{-i \lambda \alpha}, \lambda, x\right) .
\end{aligned}
$$

Hence, $m(f(t, x), \lambda, x)$ uniformly exists for $\alpha \in \mathbb{T}$. This completes the proof. $\square$ In Theorem 3.1 and Theorem 3.2, if we take $\lambda=0$, then we have

$$
a(f(t, x), 0, x)=\lim _{T \rightarrow+\infty} \frac{1}{T} \int_{t_{0}}^{t_{0}+T} f(t, x) \Delta t:=m_{t}(f(t, x))
$$

and

$$
a(f(t+\alpha), 0, x)=\lim _{T \rightarrow+\infty} \frac{1}{T} \int_{t_{0}}^{t_{0}+T} f(t+\alpha, x) \Delta t:=m_{t}(f(t+\alpha, x))
$$

uniformly converge for $x \in S$ and for $x \in S, \alpha \in \mathbb{T}$, respectively.

Definition 3.2. (3.9) and (3.10) are called the mean value and the strong mean-value of $f(t, x)$, respectively.

Lemma 3.1. Let $T \in \Pi$, then for any real number $\lambda \neq 0$,

$$
m_{t}\left(e^{i \lambda t}\right)=\lim _{T \rightarrow+\infty} \frac{1}{T} \int_{t_{0}}^{t_{0}+T} e^{i \lambda t} \Delta t=0, \text { where } t_{0} \in \mathbb{T} .
$$

Proof. First note that for any fixed $T>0$, by Lemma 3.1 and Theorem 5.2 in [36], [ $t_{0}$, $\left.t_{0}+T\right]$ contains only finitely many right scattered points. Assume that $\left[t_{0}, t_{0}+T\right]=\bigcup_{i=0}^{n}\left[\sigma\left(t_{i}\right), t_{i+1}\right]$, where 


$$
t_{0}<t_{1}<t_{2}<\cdots<t_{n}=t_{0}+T
$$

are right scattered points. Then

$$
\begin{aligned}
m_{t}\left(e^{i \lambda t}\right)= & \lim _{T \rightarrow+\infty} \frac{1}{T} \int_{t_{0}}^{t_{0}+T} e^{i \lambda t} \Delta t \\
= & \lim _{T \rightarrow+\infty} \frac{1}{T} \sum_{i=0}^{n-1}\left(\int_{t_{i}}^{\sigma\left(t_{i}\right)} e^{i \lambda t} \Delta t+\int_{\sigma\left(t_{i}\right)}^{t_{i+1}} e^{i \lambda t} \mathrm{~d} t\right) \\
= & \lim _{T \rightarrow+\infty} \frac{1}{T} \sum_{i=0}^{n-1}\left(\mu\left(t_{i}\right)\left(\cos \lambda t_{i}+i \sin \lambda t_{i}\right)+\frac{1}{\lambda}\left[\left(\sin \lambda t_{i+1}-\sin \lambda \sigma\left(t_{i}\right)\right)\right.\right. \\
& \left.\left.+i\left(\cos \lambda \sigma\left(t_{i}\right)-\cos \lambda t_{i+1}\right)\right]\right),
\end{aligned}
$$

since $\sin t$ and $\cos t$ are bounded for $t \in \mathbb{R}$, one can easily see that (3.11) holds. The proof is complete. $\square$

Theorem 3.3. Let $f(t, x) \in C\left(\mathbb{T} \times D, \mathbb{E}^{n}\right)$ be almost periodic in $t$ uniformly for $x \in D$, then for any finite set of distinct real numbers $\lambda_{1}, \lambda_{2}, \ldots, \lambda_{N}$ and any finite set of real or complex n-dimensional vectors $b_{1}, b_{2}, \ldots, b_{N}$,

$$
m_{t}\left(\left|f(t, x)-\sum_{k=1}^{N} b_{k} e^{i \lambda_{k} t}\right|^{2}\right)=m_{t}\left(|f(t, x)|^{2}\right)-\sum_{k=1}^{N}\left|a\left(f, \lambda_{k}, x\right)\right|^{2}+\sum_{k=1}^{N}\left|b_{k}-a\left(f, \lambda_{k}, x\right)\right|^{2} .
$$

Proof. Note that $|f(t, x)|^{2}=\langle f(t, x), \overline{f(t, x)}\rangle$ is almost periodic in $t$ for $x \in D$ where $\langle$,$\rangle denotes the usual inner product in \mathbb{E}^{n}$ and $\overline{f(t, x)}$ denotes the conjugate of $f(t, x)$, so it has a mean-value, thus

$$
\begin{aligned}
m_{t}\left(\left|f(t, x)-\sum_{k=1}^{N} b_{k} e^{i \lambda_{k} t}\right|^{2}\right)= & m_{t}\left(\left\langle f(t, x)-\sum_{k=1}^{N} b_{k} e^{i \lambda_{k} t}, \overline{f(t, x)}-\sum_{k=1}^{N} b_{k} e^{-i \lambda_{k} t}\right\rangle\right) \\
= & m_{t}\left(|f(t, x)|^{2}\right)-\sum_{k=1}^{N}\left\langle\overline{\left\langle b_{k}\right.} a\left(f, \lambda_{k}, x\right)\right\rangle \\
& -\sum_{k=1}^{N}\left\langle b_{k} \overline{\left.a\left(f, \lambda_{k}, x\right)\right\rangle}+\sum_{l=1}^{N} \sum_{j=1}^{N}\left\langle b_{l}, \overline{b_{j}}\right\rangle m_{t}\left(e^{i\left(\lambda_{l}-\lambda_{j}\right) t}\right),\right.
\end{aligned}
$$

by Lemma 3.1, it is easy to obtain that

$$
\begin{aligned}
m_{t}\left(\left|f(t, x)-\sum_{k=1}^{N} b_{k} e^{i \lambda_{k} t}\right|^{2}\right)= & m_{t}\left(|f(t, x)|^{2}\right)-\sum_{k=1}^{N}\left\langle\overline{b_{k}}, a\left(f, \lambda_{k}, x\right)\right\rangle \\
& -\sum_{k=1}^{N}\left\langle b_{k}, \overline{\left.a\left(f, \lambda_{k}, x\right)\right\rangle}+\sum_{j=1}^{N}\left\langle b_{j}, b_{j}\right\rangle\right. \\
= & m_{t}\left(|f(t, x)|^{2}\right)-\sum_{k=1}^{N}\left|a\left(f, \lambda_{k}, x\right)\right|^{2}+\sum_{k=1}^{N}\left|b_{k}-a\left(f, \lambda_{k}, x\right)\right|^{2} .
\end{aligned}
$$

The proof is complete.

In Theorem 3.3, if we take $b_{k}=a\left(f, \lambda_{k}, x\right)(k=1,2, \ldots, N)$, then we have the following corollary: 
Corollary 3.1. The best approximation of uniformly almost periodic function $f(t, x)$ on time scales satisfies the following:

$$
m_{t}\left(\left|f(t, x)-\sum_{k=1}^{N} b_{k} e^{i \lambda_{k} t}\right|^{2}\right)=m_{t}\left(|f(t, x)|^{2}\right)-\sum_{k=1}^{N}\left|a\left(f, \lambda_{k}, x\right)\right|^{2} .
$$

By Corollary 3.1, one can easily get the following corollary:

Corollary 3.2. Let $f(t, x) \in C\left(\mathbb{T} \times D, \mathbb{E}^{n}\right)$ be almost periodic in $t$ uniformly for $x \in D$, then

$$
\sum_{k=1}^{N}\left|a\left(f, \lambda_{k}, x\right)\right|^{2} \leq m_{t}\left(|f(t, x)|^{2}\right) .
$$

Theorem 3.4. Let $f(t, x) \in C\left(\mathbb{T} \times D, \mathbb{E}^{n}\right)$ be almost periodic in $t$ uniformly for $x \in D$, then there is a countable set of real numbers $\Lambda$ such that $a(f, \lambda, x) \equiv 0$ on $S$ if $\lambda \notin \Lambda$.

Proof. Since $f(t, x)$ is uniformly almost periodic, then for all $(t, x) \in \mathbb{T} \times S$, there exists $M>0$ such that $|f(t, x)| \leq M$. Therefore, for any $n \in \mathbb{N}$, the real number set $\left\{\lambda \in \mathbb{R}:|a(f, \lambda, x)|>\frac{1}{n}\right\} \quad$ is finite (If it is infinite, then $\sum_{k=1}^{\infty}\left|a\left(f, \lambda_{k}, x\right)\right|^{2}>\sum_{k=1}^{\infty} \frac{1}{n} \rightarrow+\infty$, this contradicts Corollary 3.2). Hence, for any fixed $x \in S$, one can obtain the real number set $\{\lambda \in \mathbb{R}: a(f, \lambda, x) \neq 0\}$ is countable. Furthermore, by Corollary 3.2, one can see that

$$
\sum_{k=1}^{N} \sup _{x \in S}\left|a\left(f, \lambda_{k}, x\right)\right|^{2} \leq M^{2}
$$

Thus, there is a countable set of real numbers $\Lambda$ such that $a(f, \lambda, x) \equiv 0$ on $S$ if $\lambda \notin$ $\Lambda$. The proof is complete.

Theorem 3.5. If $f: \mathbb{T} \times D \rightarrow \mathbb{R}^{n}$ is a non-negative almost periodic function in $t$ uniformly for $x \in D$ and $f \otimes 0$, then $a(f, 0, x)>0$.

Proof. Let $f\left(t_{0}^{\prime}, x\right)=M>0$ and pick $\delta>0$ so that $f(t, x) \geq \frac{2 M}{3}$ on $\left(t_{0}^{\prime}-\delta, t_{0}^{\prime}+\delta\right) \times S$. Let $l \in \Pi$ be an inclusion length of $E\left\{\frac{M}{3}, f, S\right\}$ and take $l>2 \delta$ (In fact, one can choose $0<\tau_{0} \in \Pi$ such that $n \tau_{0}=l \in \Pi, n$ is some positive integer). If $h$ $\in \quad \Pi, \quad t_{0} \in \mathbb{T}$, find $\tau \in E\left\{\frac{M}{3}, f, S\right\} \cap\left[h+\delta-t_{0}^{\prime}, h+\delta-t_{0}^{\prime}+l\right]$. Then $t_{0}^{\prime}-\delta+\tau \in[h, h+l]$. Either $t_{0}^{\prime}+\tau$ or $t_{0}^{\prime}-2 \delta+\tau \in[h, h+l]$ since $l>2 \delta$. In the first case if $t \in\left(t_{0}^{\prime}-\delta+\tau, t_{0}^{\prime}+\tau\right)$ then

$$
|f(t, x)| \geq|f(t+\tau, x)|-|f(t+\tau, x)-f(t, x)| \geq \frac{2 M}{3}-\frac{M}{3}=\frac{M}{3} .
$$

The second case can be handled similarly. In either case $\int_{t_{0}+h}^{t_{0}+h+l} f(t, x) \Delta t>\frac{M}{3} \delta$ since on a subinterval of length $\delta, f(t, x) \geq \frac{M}{3}$. Now write $h=(n-1) l$ to get $\int_{t_{0}+(n-1) l}^{t_{0}+n l} f(t, x) \Delta t>\frac{M}{3} \delta$. Hence

$$
\frac{1}{N l} \int_{t_{0}}^{t_{0}+N l} f(t, x) \Delta t=\frac{1}{N l} \sum_{n=1}^{N} \int_{t_{0}+(n-1) l}^{t_{0}+n l} f(t, x) \Delta t>\frac{M \delta}{3 l} .
$$

Letting $N \rightarrow \infty$ one can get $a(f, 0, x) \geq \frac{\mathrm{M} \delta}{3 l}>0$. The proof is complete. $\square$ 


\section{Pseudo almost periodic functions on time scales}

Let $B C\left(\mathbb{T} \times D, \mathbb{E}^{n}\right)$ denote the space of all bounded continuous functions from $\mathbb{T} \times D$ to $\mathbb{E}^{n}$. Set

$\mathscr{A} \mathscr{P}(\mathbb{T} \times D)_{n}=\left\{g \in C\left(\mathbb{T} \times D, \mathbb{E}^{n}\right): g\right.$ is almost periodic in $t$ uniformly for $\left.x \in D\right\}$,

$\mathscr{A} \mathscr{P}(\mathbb{T})_{n}=\left\{g \in C\left(\mathbb{T}, \mathbb{E}^{n}\right): g\right.$ is almost periodic $\}$,

$$
\begin{aligned}
\tilde{\mathscr{P}} \mathscr{A} \mathscr{P}_{0}(\mathbb{T})_{n}= & \left\{\varphi \in B C\left(\mathbb{T}, \mathbb{E}^{n}\right): \varphi \text { is } \Delta \text { - measurable such that } \lim _{r \rightarrow+\infty} \frac{1}{2 r} \int_{t_{0}-r}^{t_{0}+r}|\varphi(s)| \Delta s=0,\right. \\
& \text { where } \left.t_{0} \in \mathbb{T}, r \in \Pi\right\}
\end{aligned}
$$

and

$$
\begin{aligned}
& \tilde{\mathscr{P}} \mathscr{A}_{0} \mathscr{P}_{0}(\mathbb{T} \times D)_{n} \\
& =\left\{\varphi \in B C\left(\mathbb{T} \times D, \mathbb{E}^{n}\right): \varphi(\cdot, x) \in \tilde{\mathscr{P}} \mathscr{A} \mathscr{P}_{0}(\mathbb{T}) \text { for each } x \in D\right. \text { and } \\
& \left.\quad \lim _{r \rightarrow+\infty} \frac{1}{2 r} \int_{t_{0}-r}^{t_{0}+r}\|\varphi(s, x)\| \Delta s=0 \text { uniformly for } x \in D \text {, where } t_{0} \in \mathbb{T}, r \in \Pi\right\} .
\end{aligned}
$$

Remark 4.1. $\varphi \in \tilde{\mathscr{P}} \mathscr{A} \mathscr{P}_{0}(\mathbb{T})$ does not require $\lim _{|t| \rightarrow \infty} \varphi(t)_{\text {exists. Consider, for example, }}$ let $\mathbb{T}=\bigcup_{n=1}^{\infty}\left[n, n+\frac{1}{n}\right]$ and

$$
\varphi(t)=\left\{\begin{array}{l}
\frac{1}{\sqrt{n}}, n \leq t \leq n+\frac{1}{n}, \\
0, \quad t \text { elsewhere. }
\end{array}\right.
$$

Obviously, for any fixed $n_{0} \in \mathbb{N}$ and $t \in \mathbb{T}$, one can easily see that $t \pm n_{0} \in \mathbb{T}$, thus $n_{0}$ $\in \Pi$, that is, Tis an almost periodic time scale. It is clear that $\lim _{|t| \rightarrow \infty} \phi(t)$ does not exist, noting that $\left\{n+\frac{1}{n}\right\}_{n \in \mathbb{N}}$ are right scattered points, so

$$
\begin{aligned}
\lim _{r \rightarrow \infty} \frac{1}{2 r} \int_{t_{0}-r}^{t_{0}+r}|\varphi(s)| \Delta s & =\lim _{n \rightarrow \infty} \frac{1}{n}\left[\sum_{k=1}^{n} \int_{k}^{k+\frac{1}{k}} \frac{1}{\sqrt{k}} \mathrm{~d} s+\sum_{k=1}^{n} \mu\left(k+\frac{1}{k}\right) \frac{1}{\sqrt{k}}\right] \\
& =\lim _{n \rightarrow \infty} \frac{1}{n} \sum_{k=1}^{n}\left(\frac{1}{\sqrt{k}} \cdot \frac{1}{k}+\left(1-\frac{1}{k}\right) \frac{1}{\sqrt{k}}\right) \\
& =\lim _{n \rightarrow \infty} \frac{1}{n} \sum_{k=1}^{n} \frac{1}{\sqrt{k}}=0 .
\end{aligned}
$$

Hence $\varphi \in \tilde{\mathscr{P}} \mathscr{A} \mathscr{P}_{0}(\mathbb{T})$.

Definition 4.1. A function $f \in C\left(\mathbb{T} \times D, \mathbb{E}^{n}\right)$ is called pseudo almost periodic in $t$ uniformly for $x \in D$ if $f=g+\phi$, where $g \in \mathscr{A} \mathscr{P}(\mathbb{T} \times D)_{n}$ and $\varphi \in \tilde{\mathscr{P}} \mathscr{A} \mathscr{P}_{0}(\mathbb{T} \times D)_{n}$.

Remark 4.2. Note that $g$ and $\phi$ are uniquely determined. Indeed, since

$$
N(\varphi)=\lim _{r \rightarrow+\infty} \frac{1}{2 r} \int_{t_{0}-r}^{t_{0}+r}\|\varphi(s, x)\| \Delta s=0,
$$


if $f=g_{1}+\phi_{1}=g_{2}+\phi_{2}$, then one has $N\left(g_{1}-g_{2}\right)=0$, which implies that $g_{1}=g_{2}$, thus, $\phi_{1}=\phi_{2} . g$ and $\phi$ are called the almost periodic component and the ergodic perturbation of the function $f$, respectively. Denote by $\tilde{\mathscr{P}} \mathscr{A} \mathscr{P}(\mathbb{T} \times D)_{n}$ the set of pseudo almost periodic functions uniformly for $x \in D$.

Example 4.1. Let $\mathbb{T}=\bigcup_{k=1}^{\infty}[2 k, 2 k+1]$,

$f(t)=g(t)+\phi(t)$, where $g(t)=\sin t+\sin \pi t, \varphi(t)=-\frac{1}{t \sigma(t)}, t \in \mathbb{T}$

and

$F(t, x)=f(t) \cos x, t \in \mathbb{T}$.

Since

$$
\lim _{r \rightarrow+\infty} \frac{1}{2 r} \int_{t_{0}-r}^{t_{0}+r}|\varphi(s)| \Delta s=\lim _{r \rightarrow+\infty} \frac{1}{2 r} \int_{t_{0}-r}^{t_{0}+r} \frac{1}{s \sigma(s)} \Delta s=\left.\lim _{r \rightarrow+\infty} \frac{1}{2 r} \cdot \frac{1}{s}\right|_{t_{0}-r} ^{t_{0}+r}=0,
$$

so, $\varphi \in \tilde{\mathscr{P}} \mathscr{A} \mathscr{P}_{0}(\mathbb{T})$. Therefore, $f \in \tilde{\mathscr{P}} \mathscr{A} \mathscr{P}(\mathbb{T}), F \in \tilde{\mathscr{P}} \mathscr{A} \mathscr{P}(\mathbb{T} \times D)$.

Theorem 4.1. If $f \in \tilde{\mathscr{P}} \mathscr{A} \mathscr{P}(\mathbb{T} \times D)_{n}$, then

$$
\lim _{r \rightarrow+\infty} \frac{1}{2 r} \int_{t_{0}-r}^{t_{0}+r} f(s, x) \Delta s:=M(f)
$$

exists and is finite. It is the mean value of $f$. Moreover $M(f)=M(g)$.

Proof. Indeed

$$
\lim _{r \rightarrow+\infty} \frac{1}{2 r} \int_{t_{0}-r}^{t_{0}+r} f(s, x) \Delta s=\lim _{r \rightarrow+\infty} \frac{1}{2 r} \int_{t_{0}-r}^{t_{0}+r} g(s, x) \Delta s+\lim _{r \rightarrow+\infty} \frac{1}{2 r} \int_{t_{0}-r}^{t_{0}+r} \varphi(s, x) \Delta s .
$$

Since $g \in \mathscr{A} \mathscr{P}(\mathbb{T} \times D)_{n}$ then

$$
\lim _{r \rightarrow+\infty} \int_{t_{0}-r}^{t_{0}+r} g(s, x) \Delta s
$$

exists and is finite by Theorem 3.1. Furthermore, one has

$$
-|\varphi(s, x)| \leq \varphi(s, x) \leq|\varphi(s, x)| .
$$

Then

$$
-\lim _{r \rightarrow+\infty} \frac{1}{2 r} \int_{t_{0}-r}^{t_{0}+r}|\varphi(s, x)| \Delta s \leq \lim _{r \rightarrow+\infty} \frac{1}{2 r} \int_{t_{0}-r}^{t_{0}+r} \varphi(s, x) \Delta s \leq \lim _{r \rightarrow+\infty} \frac{1}{2 r} \int_{t_{0}-r}^{t_{0}+r} \mid \varphi(s, x) \Delta s .
$$

Since $\varphi \in \tilde{\mathscr{P}} \mathscr{A} \mathscr{P}_{0}(\mathbb{T} \times D)_{n}$,

$$
\lim _{r \rightarrow+\infty} \frac{1}{2 r} \int_{t_{0}-r}^{t_{0}+r}|\varphi(s, x)| \Delta s=0=M(\varphi) .
$$


Hence

$$
\lim _{r \rightarrow+\infty} \frac{1}{2 r} \int_{t_{0}-r}^{t_{0}+r} \varphi(s, x) \Delta s=0 .
$$

Therefore $M(f)=M(g)$. The proof is complete. $\square$

By Definition 4.1 and the definition of $a(\cdot, \lambda, x)$, one can easily have

Corollary 4.1. If $f \in \tilde{\mathscr{P}} \mathscr{A} \mathscr{P}(\mathbb{T} \times D)_{n}$ then $a(f, \lambda, x)=a(g, \lambda, x)$.

Furthermore, from Definition 4.1, one can easily show that

Theorem 4.2. If $f \in \tilde{\mathscr{P}} \mathscr{A} \mathscr{P}(\mathbb{T} \times D)_{n}$ and $g$ is the almost periodic component of $f$, then we have

$$
g(\mathbb{T} \times S) \subset \overline{f(\mathbb{T} \times S)}
$$

and

$$
\|f\| \geq\|g\| \geq \inf _{(t, x) \in \mathbb{T} \times S}|g(t, x)| \geq \inf _{(t, x) \in \mathbb{T} \times S}|f(t, x)|,
$$

where $f(\mathbb{T} \times S)$ and $g(\mathbb{T} \times S)$ denote the value field of $f$ and $g$ on $\mathbb{T} \times S$, respectively, $\overline{f(\mathbb{T} \times S)}$ denotes the closure of $f(\mathbb{T} \times S)$, where $S$ is an arbitrary compact subset of $D$.

Definition 4.2. A closed subset $C$ of $\mathbb{T}$ is said to be an ergodic zero set in $\mathbb{T}$ if

$$
\frac{\mu_{\Delta}\left(C \cap\left(\left[t_{0}-r, t_{0}+r\right] \cap \mathbb{T}\right)\right)}{2 r} \rightarrow 0 \text { as } r \rightarrow \infty, \text { where }_{0} \in \mathbb{T} .
$$

By the definition of $\tilde{\mathscr{P}} \mathscr{A} \mathscr{P}_{0}(\mathbb{T} \times D)_{n}$, the proof of the following theorem is straightforward.

Theorem 4.3. A function $\varphi \in \tilde{\mathscr{P}} \mathscr{A} \mathscr{P}_{0}(\mathbb{T} \times D)_{n}$ if and only if for $\varepsilon>0$, the set $C_{\varepsilon}=\{t \in \mathbb{T}:|\varphi(t, x)| \geq \varepsilon\}$ is an ergodic zero subset in $\mathbb{T}$.

Theorem 4.4. (i) A function $\varphi \in \tilde{\mathscr{P}} \mathscr{A} \mathscr{P}_{0}(\mathbb{T} \times D)$ if and only if $|\varphi|^{2} \in \tilde{\mathscr{P}} \mathscr{A} \mathscr{P}_{0}(\mathbb{T} \times D)$.

(ii) $\Phi \in \tilde{\mathscr{P}} \mathscr{A} \mathscr{P}_{0}(\mathbb{T} \times D)_{n}$ if and only if the norm function $|\Phi(\cdot, x)| \in \tilde{\mathscr{P}} \mathscr{A} \mathscr{P}_{0}(\mathbb{T} \times D)$.

Proof. (i) The sufficiency follows since

$$
\begin{aligned}
\frac{1}{2 r} \int_{t_{0}-r}^{t_{0}+r}|\varphi(s, x)| \Delta s & \leq \frac{1}{2 r}\left[\int_{t_{0}-r}^{t_{0}+r}|\varphi(t, x)|^{2} \Delta s\right]^{1 / 2}\left[\int_{t_{0}-r}^{t_{0}+r} 1 \Delta s\right]^{1 / 2} \\
& =\left[\frac{1}{2 r} \int_{t_{0}-r}^{t_{0}+r}|\varphi(s, x)|^{2} \Delta s\right]^{1 / 2} .
\end{aligned}
$$

The necessity follows from the fact that

$$
\frac{1}{2 r} \int_{t_{0}-r}^{t_{0}+r}|\varphi(s, x)|^{2} \Delta s \leq\|\varphi\| \frac{1}{2 r} \int_{t_{0}-r}^{t_{0}+r}|\varphi(t, x)| \Delta s,
$$

since $\phi$ is bounded on $\mathbb{T}$. Therefore, one can easily see that $(i)$ is satisfied. 
(ii) By $\quad(i), \quad \Phi=\left(\varphi_{1}, \varphi_{2}, \ldots, \varphi_{n}\right) \in \mathscr{P} \mathscr{A} \mathscr{P}_{0}(\mathbb{T} \times D)_{n} \quad$ if and only if $\varphi_{i} \bar{\varphi}_{i} \in \tilde{\mathscr{P}} \mathscr{A} \mathscr{P}_{0}(\mathbb{T} \times D), i=1,2, \ldots, n$. The latter is equivalent to $|\Phi(\cdot, x)|^{2}=\sum_{i=1}^{n}|\varphi(\cdot, x)|^{2} \in \tilde{\mathscr{P}} \mathscr{A} \mathscr{P}_{0}(\mathbb{T} \times D)$, which again by $(i)$, is equivalent to $|\Phi(\cdot, x)| \in \tilde{\mathscr{P}} \mathscr{A} \mathscr{P}_{0}(\mathbb{T} \times D)$.

The proof is complete.

For $H=\left(h_{1}, h_{2}, \ldots, h_{n}\right) \in \mathbb{E}^{n}$, suppose that $H(t) \in D$ for all $t \in \mathbb{T}$. Define $H \times \iota: \mathbb{T} \rightarrow \mathbb{T} \times D$ by

$$
H \times \iota(t)=\left(t, h_{1}(t), h_{2}(t), \ldots, h_{n}(t)\right) .
$$

For $F=\left(f_{1}, f_{2}, \ldots, f_{n}\right) \in \tilde{\mathscr{P}} \mathscr{A} \mathscr{P}(\mathbb{T} \times D)_{n}$, let $G=\left(g_{1}, g_{2}, \ldots, g_{n}\right)$ and $\Phi=\left(\phi_{1}, \phi_{2}, \ldots\right.$, $\left.\phi_{n}\right)$, where $g_{i}$ and $\phi_{i}$ are the almost periodic component and the ergodic perturbation of $f_{i}(i=1,2, \ldots, n)$, respectively.

Definition 4.3. Let $S$ be a compact subset of $D$. A function $f \in C\left(\mathbb{T} \times D, \mathbb{E}^{n}\right)$ is said to be continuous in $x \in S$ uniformly for $t \in \mathbb{T}$ if for given $x \in S$ and $\varepsilon>0$, there exists a $\delta(x$, $\varepsilon)>0$ such that $x^{\prime} \in S$ and $\left|x-x^{\prime}\right|<\delta(x, \varepsilon)$ imply that $\left|f\left(t, x^{\prime}\right)-f(t, x)\right|<\varepsilon$ for all $t \in \mathbb{T}$.

Theorem 4.5. Suppose that the function $f \in \tilde{\mathscr{P}} \mathscr{A} \mathscr{P}(\mathbb{T} \times D)_{n}$ is continuous in $x \in S$ uniformly for $t \in \mathbb{T}$ and $F \in \tilde{\mathscr{P}} \mathscr{A} \mathscr{P}(\mathbb{T})_{n}$ such that $F(\mathbb{T}) \subset D$, then $f \circ(F \times \iota) \in \tilde{\mathscr{P}} \mathscr{A} \mathscr{P}(\mathbb{T})_{n}$, where $F(\mathbb{T})$ denotes the value field of $F$ and $S$ is an arbitrary compact subset of $D$.

Proof. Let $f=g+\phi$ and $F=G+\Phi$ with $G=\left(g_{1}, g_{2}, \ldots, g_{n}\right) \in \mathscr{A} \mathscr{P}(\mathbb{T})_{n}$ as above. Note that

$$
\begin{aligned}
f \circ(F \times \iota) & =g \circ(F \times \iota)+\varphi \circ(F \times \iota) \\
& =g \circ(G \times \iota)+[g \circ(F \times \iota)-g \circ(G \times \iota)+\varphi \circ(F \times \iota)] .
\end{aligned}
$$

It follows from Theorem 4.2 that $G(\mathbb{T}) \subset \overline{F(\mathbb{T})} \subset D$. By Theorem 3.15 in [35], we have $g \circ(G \times \iota) \in \mathscr{A} \mathscr{P}(\mathbb{T})_{n}$. To finish the proof, we need to show that the function $h$ $=g$ o $(F \times \iota)-g$ o $(G \times \iota)+\phi$ o $(F \times \iota)$ is in $\tilde{\mathscr{P}} \mathscr{A} \mathscr{P}_{0}(\mathbb{T})_{n}$.

First we show that $g \circ(F \times \iota)-g \circ(G \times \iota) \in \tilde{\mathscr{P}} \mathscr{A} \mathscr{P}_{0}(\mathbb{T})_{n}$.

It is trivial in the case that $g=0$. So we assume that $g \neq 0$. Set $D_{1}=\overline{F(\mathbb{T})}$. By Theorem 3.1 in [35], the function $g$ is uniformly continuous on $\mathbb{T} \times D_{1}$. For $\varepsilon>0$, there exists a $\delta>0$ such that

$$
\left|g\left(t, x_{1}\right)-g\left(t, x_{2}\right)\right|<\frac{\varepsilon}{2}, x_{1}, x_{2} \in D_{1},\left|x_{1}-x_{2}\right|<\delta, t \in \mathbb{T}
$$

Set

$$
C_{\delta}=\{t \in \mathbb{T}:|F(t)-G(t)|=|\Phi(t)| \geq \delta\}
$$

It follows from Theorem 4.3 and $(i i)$ of Theorem 4.4 that $C_{\delta}$ is an ergodic zero subset of $\mathbb{T}$. We can find $T>0$ such that when $r \geq T$,

$$
\frac{\mu_{\Delta}\left(\left(\left[t_{0}-r, t_{0}+r\right] \cap \mathbb{T}\right) \cap C_{\delta}\right)}{2 r}<\frac{\varepsilon}{4\|g\|} .
$$


By (4.1), (4.2) and (4.3), we have

$$
\begin{aligned}
& \frac{1}{2 r} \int_{t_{0}-r}^{t_{0}+r}|g(s, F(s))-g(s, G(s))| \Delta s \\
& =\frac{1}{2 r}\left\{\int_{\left(\left[t_{0}-r, t_{0}+r\right] \cap \mathbb{T}\right) \backslash C_{\delta}}+\int_{\left(\left[t_{0}-r, t_{0}+r\right] \cap T\right) \cap C_{\delta}}|g(s, F(s))-g(s, G(s))| \Delta s\right\} \\
& \leq \frac{\varepsilon}{2}+2\|g\| \frac{\mu_{\Delta}\left(\left(\left[t_{0}-r, t_{0}+r\right] \cap \mathbb{T}\right) \cap C_{\delta}\right)}{2 r}<\varepsilon .
\end{aligned}
$$

Therefore, $g \circ(F \times \iota)-g \circ(G \times \iota) \in \tilde{\mathscr{P}} \mathscr{A} \mathscr{P}_{0}(\mathbb{T})_{n}$.

Finally, we show that $\varphi \circ(F \times \iota) \in \tilde{\mathscr{P}} \mathscr{A} \mathscr{P}_{0}(\mathbb{T})_{n}$. Note that $f=g+\phi$ and $g$ is uniformly continuous on $\mathbb{T} \times D_{1}$. By the hypothesis, $f$ is continuous in $S \subset D_{1}$ uniformly for $t \in \mathbb{T}$; so is $\phi$. Since $D_{1}$ is compact in $\mathbb{E}^{n}$, one can find, say $m$, open balls $O_{k}$ with center $x^{k} \in D_{1}, k=1,2, \ldots, m$, and radius $\delta\left(x^{k}, \varepsilon / 2\right)$ such that $D_{1} \subset \bigcup_{k=1}^{m} O_{k}$ and

$$
\left|\varphi(t, x)-\varphi\left(t, x^{k}\right)\right|<\frac{\varepsilon}{2}, x \in O_{k}, t \in \mathbb{T} .
$$

The set

$$
B_{k}=\left\{t \in \mathbb{T}: F(t) \in O_{k}\right\}
$$

is open and $\mathbb{T}=\bigcup_{k=1}^{m} B_{k}$. Let $E_{k}=B_{k} \backslash \bigcup_{j=1}^{k-1} B_{j}$, then $E_{k} \cap E_{j}=\varnothing$ when $k \neq j, 1 \leq k, j$ $\leq m$.

Since for each $\varphi\left(\cdot, x^{(k)}\right) \in \tilde{\mathscr{P}} \mathscr{A} \mathscr{P}_{0}(\mathbb{T})_{n}$, there is a number $T_{0}>0$ such that

$$
\sum_{k=1}^{m} \frac{1}{2 r} \int_{t_{0}-r}^{t_{0}+r}\left|\varphi\left(s, x^{(k)}\right)\right| \Delta s<\frac{\varepsilon}{2}, r \geq T_{0} .
$$

It follows from (4.4), (4.5) and (4.6) that

$$
\begin{aligned}
\frac{1}{2 r} \int_{t_{0}-r}^{t_{0}+r}|\varphi(s, F(s))| \Delta s & \leq \frac{1}{2 r} \sum_{k=1}^{m} \int_{E_{k} \cap\left(\left[t_{0}-r, t_{0}+r\right] \cap \mathbb{T}\right)}\left(\left|\varphi(s, F(s))-\varphi\left(s, x^{(k)}\right)\right|+\mid \varphi\left(s, x^{(k)} \mid\right)+\Delta s\right. \\
& \leq \frac{\varepsilon}{2}+\sum_{k=1}^{m} \frac{1}{2 r} \int_{t_{0}-r}^{t_{0}+r}\left|\varphi\left(s, x^{(k)}\right)\right| \Delta s<\varepsilon .
\end{aligned}
$$

This shows that $\varphi \circ(F \times \iota) \in \tilde{\mathscr{P}} \mathscr{A} \mathscr{P}_{0}(\mathbb{T})_{n}$. The proof is complete. $\square$

Define

$$
\begin{aligned}
& \mathbb{E}_{0}(\mathbb{T} \times D)_{n}=\left\{f \in C\left(\mathbb{T} \times D, \mathbb{E}^{n}\right): f(t, x) \rightarrow 0, \text { uniformly in } x \in D, \text { as }|t| \rightarrow \infty\right\} . \\
& \mathbb{E}_{0}(\mathbb{T})_{n}=\left\{f \in C\left(\mathbb{T}, \mathbb{E}^{n}\right): f(t) \rightarrow 0, \text { as }|t| \rightarrow \infty\right\} .
\end{aligned}
$$

Definition 4.4. Let $\mathscr{A} \mathscr{A} \mathscr{P}(\mathbb{T} \times D)_{n}$ denote all the functions $f$ of the form $f=g+\phi$, where $g \in \mathscr{A} \mathscr{P}(\mathbb{T} \times D)_{n}$ and $\varphi \in E_{0}(\mathbb{T} \times D)_{n}$. The members of $\mathscr{A} \mathscr{A} \mathscr{P}(\mathbb{T} \times D)_{n}$ are called asymptotically almost periodic functions in $t$ uniformly for $x \in D$.

It is obvious that $E_{0}(\mathbb{T} \times D)_{n} \subset \tilde{\mathscr{P}} \mathscr{A} \mathscr{P}_{0}(\mathbb{T} \times D)_{n} \quad$ and 
$\mathscr{A} \mathscr{A} \mathscr{P}(\mathbb{T} \times D)_{n} \subset \tilde{\mathscr{P}} \mathscr{A} \mathscr{P}(\mathbb{T} \times D)_{n}$.

Corollary 4.2. If $f \in \mathscr{A} \mathscr{A} \mathscr{P}(\mathbb{T} \times D)_{n}$ and $F \in \mathscr{A} \mathscr{A} \mathscr{P}(\mathbb{T})_{n}$ such that $F(\mathbb{T}) \subset D$, then $f \circ(F \times \iota) \in \mathscr{A} \mathscr{A} \mathscr{P}(\mathbb{T})_{n}$.

Proof. Obliviously,

$$
\begin{aligned}
f \circ(F \times \iota) & =g \circ(F \times \iota)+\varphi \circ(F \times \iota) \\
& =g \circ(G \times \iota)+[g \circ(F \times \iota)-g \circ(G \times \iota)+\varphi \circ(F \times \iota)],
\end{aligned}
$$

where $g \circ(G \times \imath) \in \mathscr{A} \mathscr{P}(\mathbb{T})_{n}$. By the hypothesis that $\Phi=F-G \in E_{0}(\mathbb{T})_{n}$ and $\varphi \in E_{0}(\mathbb{T} \times D)_{n}$, it follows that $g \circ(F \times \iota)-g \circ(G \times \iota) \in E_{0}(\mathbb{T})_{n}$ since the uniform continuity of $g$ and $\varphi \circ(F \times \iota) \in E_{0}(\mathbb{T})_{n}$ since $\varphi(t, F(t)) \leq \sup _{x \in D} \varphi(t, x)$. The proof is complete. $\square$

Theorem 4.6. Suppose that $g \in \mathscr{A} \mathscr{P}(\mathbb{T} \times D)_{n}$ satisfies that for every $\varepsilon>0$,

$$
\frac{\mu_{\Delta}\left\{t: g(t, x)>-\varepsilon, t \in\left[t_{0}-r, t_{0}+r\right] \cap \mathbb{T}\right\}}{2 r} \rightarrow 1 \text {, as } r \rightarrow+\infty \text {, where } t_{0} \in \mathbb{T}, r \in \Pi .
$$

Then $g \geq 0$ for all $\mathbb{T} \times S$, where $S$ is an arbitrary compact subset of $D$.

Proof. Suppose that the conclusion does not hold. This implies that $g\left(t_{0}^{\prime}, x\right)<0$ for some $t_{0}^{\prime}$. Choose $\varepsilon>0, \varepsilon<-g\left(t_{0}^{\prime}, x\right)$.

By continuity, there exists $\delta>0$ so that $\left|t-t^{\prime}{ }_{0}\right| \leq \delta$ implies $g(t, x)<-\varepsilon$. In view of Definition 2.4, there exists $l(\varepsilon, S)>0$ so that in each interval $I$ of length $l$, one can find $\frac{\varepsilon}{2}$-almost period $\tau$ with the property that

$$
|g(t+\tau, x)-g(t, x)|<\frac{\varepsilon}{2} .
$$

Choose a sequence $\tau_{k}$ of almost periods, $\tau_{k} \in\left[t_{0}+k l, t_{0}+(k+1) l\right]$, we have

$$
g\left(t+\tau_{k}, x\right)<-\frac{\varepsilon}{2}, \text { and } t \in\left[t_{0}^{\prime}-\delta, t_{0}^{\prime}+\delta\right] \cap \mathbb{T} \text { and every } k \in \mathbb{N} .
$$

Denote $M=\left|t_{0}^{\prime}\right|+\delta$, we have

$$
\mu_{\Delta}\left\{t \in\left[t_{0}-k l-M, t_{0}+k l+M\right] \cap \mathbb{T}: g(t, x)<-\frac{\varepsilon}{2}\right\} \geq 2 k \delta .
$$

Therefore,

$$
\frac{\mu_{\Delta}\left\{t \in\left[t_{0}-k l-M, t_{0}+k l+M\right] \cap \mathbb{T}: g(t, x)<-\frac{\varepsilon}{2}\right\}}{2 k l+2 M} \geq \frac{2 k \delta}{2 k l+2 M} .
$$

The right hand side does not tend to zero as $k \rightarrow+\infty$. This contradicts the assumption made in the lemma. Therefore, $g \geq 0$. The proof is complete. $\square$

Theorem 4.7. If $f \in C\left(\mathbb{T} \times D, \mathbb{E}^{n}\right), f=g+\varphi$, where $g \in \mathscr{A} \mathscr{P}(\mathbb{T} \times D)_{n}$ and $\varphi \in \tilde{\mathscr{P}} \mathscr{A} \mathscr{P}_{0}(\mathbb{T} \times D)_{n}$, then

(i) If $\lim _{|t| \rightarrow \infty} \varphi(t, x)_{\text {exists, then }} \lim _{|t| \rightarrow \infty} \varphi(t, x)=0$.

(ii) For all $(t, x) \in \mathbb{T} \times S$, if $f \geq 0$ then $g \geq 0$, where $S$ is an arbitrary compact subset of $D$.

Proof. (i) Suppose that the property does not hold, then there exist a constant $\tilde{\alpha}>0$ and $c \in \Pi$ such that $\varphi(t, x)>\tilde{\alpha}$ for $t \geq c$, which yields 


$$
\frac{1}{r} \int_{t_{0}}^{t_{0}+r}|\varphi(s, x)| \Delta s=\frac{1}{r}\left[\int_{t_{0}}^{t_{0}+c}|\varphi(s, x)| \Delta s+\int_{t_{0}+c}^{t_{0}+r}|\varphi(s, x)| \Delta s\right] \geq \frac{1}{r} \tilde{\alpha}(r-c) .
$$

Passing to the limit as $r \rightarrow \infty$, we obtain

$$
\lim _{r \rightarrow \infty} \frac{1}{2 r} \int_{t_{0}-r}^{t_{0}+r}|\varphi(s, x)| \Delta s \geq \tilde{\alpha},
$$

which contradicts the fact that $\varphi \in \tilde{\mathscr{P}} \mathscr{A} \mathscr{P}_{0}(\mathbb{T} \times D)_{n}$.

(ii) Assuming $f \geq 0$, we want to show that $g \geq 0$. We have $f=g+\phi$ with

$$
\lim _{r \rightarrow \infty} \frac{1}{2 r} \int_{t_{0}-r}^{t_{0}+r}|\varphi(s, x)| \Delta s=0
$$

Thus, there exists $\left\{c_{n}\right\}_{n \in \mathbb{N}} \subset \Pi, c_{n} \rightarrow+\infty$ as $n \rightarrow \infty$ such that $g\left(t+c_{n}, x\right) \rightarrow g(t, x)$ for all $(t, x) \in \mathbb{T} \times S$. Furthermore, for any $\varepsilon>0$ and $r>0$, one can easily get

$$
\mu_{\Delta}\left\{t \in\left[t_{0}-r, t_{0}+r\right] \cap \mathbb{T}: \varphi(t, x)>\varepsilon\right\} \rightarrow 0, \text { as } r \rightarrow \infty,
$$

which implies that

$$
\frac{\mu_{\Delta}\left\{t: g(t, x)>-\varepsilon, t \in\left[t_{0}-r, t_{0}+r\right] \cap \mathbb{T}\right\}}{2 r} \rightarrow 1, \text { as } r \rightarrow+\infty, \text { where } t_{0} \in \mathbb{T}, r \in \Pi .
$$

By Theorem 4.6, one can have $g(t, x) \geq 0$ for all $(t, x) \in \mathbb{T} \times S$.

The proof is complete.

\section{Pseudo almost periodic solutions of dynamic equations on time scales}

Consider the non-autonomous equation

$$
x^{\Delta}=A(t) x+F(t)
$$

and its associated homogeneous equation

$$
x^{\Delta}=A(t) x,
$$

where the $n \times n$ coefficient matrix $A(t)$ is continuous on $\mathbb{T}$ and column vector $F=$ $\left(f_{1}, f_{2}, \ldots, f_{n}\right)^{T}$ is in $\mathbb{E}^{n}$. Define $\|F\|=\sup _{t \in \mathbb{T}}|F(t)|$. We will call $A(t)$ almost periodic if all the entries are almost periodic.

Definition 5.1 ([37]). Equation (5.2) is said to admit an exponential dichotomy on Tif there exist positive constants $K, \alpha$, projection $P$ and the fundamental solution matrix $X(t)$ of (5.2), satisfying

$$
\left\{\begin{array}{l}
\left|X(t) P X^{-1}(s)\right| \leq K e_{\ominus \alpha}(t, s), s, t \in \mathbb{T}, t \geq s, \\
\left|X(t)(I-P) X^{-1}(s)\right| \leq K e_{\ominus \alpha}(s, t), s, t \in \mathbb{T}, t \leq s .
\end{array}\right.
$$

Lemma 5.1. Let $\alpha>0$, then for any fixed $s \in \mathbb{T}$ and $s=-\infty$, one has the following:

$$
e_{\ominus \alpha}(t, s) \rightarrow 0, t \rightarrow+\infty \text {. }
$$

Proof. If $\mu(t)>0$, since $\alpha \in \mathcal{R}^{+}$, we have

$$
1+\mu(t) \ominus \alpha=1+\mu(t) \frac{-\alpha}{1+\mu(t) \alpha}=\frac{1}{1+\mu(t) \alpha}<1 .
$$


Thus, $\ominus \alpha \in \mathcal{R}^{+}$and it is easy to have

$$
\log (1+\mu(t) \ominus \alpha) \in \mathbb{R} \text { for all } t \in \mathbb{T} .
$$

So

$$
\xi_{\mu(t)}(\ominus \alpha)=\frac{\log (1+\mu(t) \ominus \alpha)}{\mu(t)}<0 .
$$

Hence

$$
e_{\ominus \alpha}(t, s)=\exp \left\{\int_{s}^{t} \xi_{\mu(t)}(\ominus \alpha) \Delta t\right\} \rightarrow 0 \text { as } t \rightarrow+\infty .
$$

If $\mu(t)=0$, one can easily get the conclusion. If $s=-\infty$, it is easy to see that $\int_{s}^{t} \xi_{\mu(t)}(\ominus \alpha) \Delta t \rightarrow-\infty$ as $t \rightarrow+\infty$, thus, $e_{\ominus \alpha}(t, s) \rightarrow 0$. The proof is complete. $\square$

Theorem 5.1. Suppose that $A(t)$ is almost periodic, (5.2) admits an exponential dichotomy and the function $F \in \tilde{\mathscr{P}} \mathscr{A} \mathscr{P}_{0}(\mathbb{T})_{n}$. Then (5.1) has a unique bounded solution $x \in \tilde{\mathscr{P}} \mathscr{A} \mathscr{P}_{0}(\mathbb{T})_{n}$.

Proof. Similar to the proof of Theorem 4.1 in [35], by checking directly, one can see that the function:

$$
x(t)=\int_{-\infty}^{t} X(t) P X^{-1}(\sigma(s)) F(s) \Delta s-\int_{t}^{+\infty} X(t)(I-P) X^{-1}(\sigma(s)) F(s) \Delta s
$$

is a solution of (5.1). Now, we show that the solution is bounded. It follows from (5.4) that

$$
\begin{aligned}
|x(t)| & =\sup _{t \in \mathbb{T}}\left|\int_{-\infty}^{t} X(t) P X^{-1}(\sigma(s)) F(s) \Delta s-\int_{t}^{+\infty} X(t)(I-P) X^{-1}(\sigma(s)) F(s) \Delta s\right| \\
& \leq \sup _{t \in \mathbb{T}}\left(\left|\int_{-\infty}^{t} e_{\ominus \alpha}(t, \sigma(s)) \Delta s\right|+\left|\int_{t}^{+\infty} e_{\ominus \alpha}(\sigma(s), t) \Delta s\right|\right) K\|F\| \\
& \leq\left(\frac{1}{\alpha}-\frac{1}{\ominus \alpha}\right) K\|F\|=\frac{2+\mu \alpha}{\alpha} K\|F\|,
\end{aligned}
$$

where $\|\cdot\|=\sup _{t \in \mathbb{T}}|\cdot|$. The solution $x$ is bounded since $F$ is bounded. By Lemma 4.13 in [35], the bounded solution is unique since the homogeneous equation (5.2) has no nontrivial bounded solution.

In the following, we show that $x \in \tilde{\mathscr{P}} \mathscr{A} \mathscr{P}_{0}(\mathbb{T})_{n}$. Let $I(t)=\int_{-\infty}^{t} X(t) P X^{-1}(\sigma(s)) F(s) \Delta s$ and $H(t)=\int_{t}^{+\infty} X(t)(I-P) X^{-1}(\sigma(s)) F(s) \Delta s$. Then $x=I+H$. It follows from (5.3) and Theorem 2.15 in [38] that 


$$
\begin{aligned}
\frac{1}{2 r} \int_{t_{0}-r}^{t_{0}+r}|I(t)| \Delta t & \leq \frac{1}{2 r} \int_{t_{0}-r}^{t_{0}+r} \Delta t \int_{-\infty}^{t}\left|X(t) P X^{-1}(\sigma(s))\right||F(s)| \Delta s \\
& \leq \frac{1}{2 r} \int_{t_{0}-r}^{t_{0}+r} \Delta t \int_{-\infty}^{t} K e_{\ominus \alpha}(t, \sigma(s))|F(s)| \Delta s \\
& =\frac{1}{2 r} \int_{t_{0}-r}^{t_{0}+r} \Delta t\left(\int_{-\infty}^{t_{0}-r}+\int_{t_{0}-r}^{t} K e_{\ominus \alpha}(t, \sigma(s))|F(s)|\right) \Delta s \\
& =\frac{1}{2 r} \int_{-\infty}^{t_{0}-r}|F(s)| \Delta s \int_{t_{0}-r}^{t_{0}+r} K e_{\ominus \alpha}(t, \sigma(s)) \Delta t \\
& +\frac{1}{2 r} \int_{t_{0}-r}^{t_{0}+r}|F(s)| \Delta s \int_{s}^{t_{0}+r} K e_{\ominus \alpha}(t, \sigma(s)) \Delta t=I_{1}+I_{2} .
\end{aligned}
$$

To show that $I \in \tilde{\mathscr{P}} \mathscr{A} \mathscr{P}_{0}(\mathbb{T})_{n}$, we only need to show that both $I_{1} \rightarrow 0$ and $I_{2} \rightarrow 0$ when $r \rightarrow \infty$. By Lemma 5.1, one can obtain

$$
\begin{aligned}
& I_{1}=\frac{1}{2 r} \int_{-\infty}^{t_{0}-r}|F(s)| \Delta s \int_{t_{0}-r}^{t_{0}+r} K e_{\ominus \alpha}(t, \sigma(s)) \Delta t \\
& =\frac{1}{2 r} \int_{-\infty}^{t_{0}-r}|F(s)| \Delta s \int_{t_{0}-r}^{t_{0}+r} \frac{K}{1+\mu(t) \ominus \alpha} e_{\ominus \alpha}(\sigma(t), \sigma(s)) \Delta t \\
& \leq \frac{1}{2 r} K(1+\bar{\mu} \alpha) \int_{-\infty}^{t_{0}-r}|F(s)| \Delta s \int_{t_{0}-r}^{t_{0}+r} e_{\alpha}(\sigma(s), \sigma(t)) \Delta t \\
& =\frac{1}{2 r} \frac{K(1+\bar{\mu} \alpha)}{\alpha} \int_{-\infty}^{t_{0}-r}|F(s)|\left[e_{\alpha}\left(\sigma(s), t_{0}-r\right)-e_{\alpha}\left(\sigma(s), t_{0}+r\right)\right] \Delta s \\
& \leq \frac{1}{2 r} \frac{K(1+\bar{\mu} \alpha)}{\alpha}\|F\|\left(\int_{-\infty}^{t_{0}-r} e_{\ominus \alpha}\left(t_{0}-r, \sigma(s)\right) \Delta s-\int_{-\infty}^{t_{0}-r} e_{\ominus \alpha}\left(t_{0}+r, \sigma(s)\right) \Delta s\right) \\
& =\frac{1}{2 r} \frac{K(1+\bar{\mu} \alpha)}{\alpha} \frac{1}{\ominus \alpha}\left(e_{\ominus \alpha}\left(t_{0}-r,-\infty\right)-e_{\ominus \alpha}\left(t_{0}-r, t_{0}-r\right)-e_{\ominus \alpha}\left(t_{0}+r,-\infty\right)\right. \\
& \left.+e_{\ominus \alpha}\left(t_{0}+r, t_{0}-r\right)\right) \rightarrow 0 \text { as } r \rightarrow+\infty \\
& I_{2}=\frac{1}{2 r} \int_{t_{0}-r}^{t_{0}+r}|F(s)| \Delta s \int_{s}^{t_{0}+r} K e_{\ominus \alpha}(t, \sigma(s)) \Delta t \\
& =\frac{1}{2 r} \int_{t_{0}-r}^{t_{0}+r}|F(s)| \Delta s \int_{s}^{t_{0}+r} \frac{K}{1+\mu(t) \ominus \alpha} e_{\ominus \alpha}(\sigma(t), \sigma(s)) \Delta t \\
& \leq \frac{1}{2 r} K(1+\bar{\mu} \alpha) \int_{t_{0}-r}^{t_{0}+r}|F(s)| \Delta s \int_{s}^{t_{0}+r} e_{\alpha}(\sigma(s), \sigma(t)) \Delta t \\
& =\frac{1}{2 r} \frac{K(1+\bar{\mu} \alpha)}{\alpha} \int_{t_{0}-r}^{t_{0}+r}|F(s)|\left[e_{\alpha}(\sigma(s), s)-e_{\alpha}\left(\sigma(s), t_{0}+r\right)\right] \Delta s \\
& \leq \frac{1}{2 r} \frac{K(1+\bar{\mu} \alpha)^{2}}{\alpha} \int_{t_{0}-r}^{t_{0}+r}|F(s)| \Delta s
\end{aligned}
$$


Therefore, by $(i i)$ of Theorem $4.4,|F(\cdot)| \in \tilde{\mathscr{P}} \mathscr{A} \mathscr{P}_{0}(\mathbb{T})$, so $I_{2} \rightarrow 0$ as $r \rightarrow+\infty$.

Similarly, one can show that $H \in \tilde{\mathscr{P}} \mathscr{A} \mathscr{P}_{0}(\mathbb{T})_{n}$. The proof is complete. $\square$

Theorem 5.2. Suppose that $A(t)$ is almost periodic and (5.2) admits an exponential dichotomy. Then, for every $F \in \tilde{\mathscr{P}} \mathscr{A} \mathscr{P}(\mathbb{T})_{n}$. (5.1) has a unique bounded solution $x_{F} \in \mathscr{P} \mathscr{A} \mathscr{P}(\mathbb{T})_{n}$. The mapping $F \rightarrow x_{F}$ is bounded and linear with

$$
\left\|x_{F}\right\| \leq\left(\frac{1}{\alpha}-\frac{1}{\ominus \alpha}\right) K\|F\|=\frac{2+\mu \alpha}{\alpha} K\|F\| .
$$

Proof. Since $F \in \tilde{\mathscr{P}} \mathscr{A} \mathscr{P}(\mathbb{T})_{n}, F=G+\Phi$, where $G \in \mathscr{A} \mathscr{P}(\mathbb{T})_{n}$ and $\Phi \in \tilde{\mathscr{P}} \mathscr{A} \mathscr{P} 0(\mathbb{T})_{n}$. According to the proof of Theorem 5.1, the function

$$
\begin{aligned}
x_{F}= & \int_{-\infty}^{t} X(t) P X^{-1}(\sigma(s)) F(s) \Delta s-\int_{t}^{+\infty} X(t)(I-P) X^{-1}(\sigma(s)) F(s) \Delta s \\
= & \left(\int_{-\infty}^{t} X(t) P X^{-1}(\sigma(s)) G(s) \Delta s-\int_{t}^{+\infty} X(t)(I-P) X^{-1}(\sigma(s)) G(s) \Delta s\right) \\
& +\left(\int_{-\infty}^{t} X(t) P X^{-1}(\sigma(s)) \Phi(s) \Delta s-\int_{t}^{+\infty} X(t)(I-P) X^{-1}(\sigma(s)) \Phi(s) \Delta s\right) \\
:= & x_{G}+x_{\Phi}
\end{aligned}
$$

is the unique solution of (5.1), where

$$
\begin{aligned}
x_{G}:= & \int_{-\infty}^{t} X(t) P X^{-1}(\sigma(s)) G(s) \Delta s-\int_{t}^{+\infty} X(t)(I-P) X^{-1}(\sigma(s)) G(s) \Delta s, \\
x_{\Phi}: & \int_{-\infty}^{t} X(t) P X^{-1}(\sigma(s)) \Phi(s) \Delta s-\int_{t}^{+\infty} X(t)(I-P) X^{-1}(\sigma(s)) \Phi(s) \Delta s .
\end{aligned}
$$

By Theorem 4.1 in [35], $x_{G} \in \mathscr{A} \mathscr{P}(\mathbb{T})_{n}$. By Theorem 5.1, $x_{\Phi} \in \tilde{\mathscr{P}} \mathscr{A} \mathscr{P}_{0}(\mathbb{T})_{n}$. Therefore, $x_{F} \in \tilde{\mathscr{P}} \mathscr{A} \mathscr{P}(\mathbb{T})_{n}$. Obviously, the mapping $F \rightarrow x_{F}$ is linear. The proof is complete. $\square$

Lemma 5.2. Let $c_{i}(t): \mathbb{T} \rightarrow \mathbb{R}^{+}$be an almost periodic function, $-c_{i} \in \mathcal{R}^{+}, T \in \Pi$ and

$$
m\left(c_{i}\right)=\lim _{T \rightarrow \infty} \frac{1}{T} \int_{t}^{t+T} c_{i}(s) \Delta s>0, \quad i=1,2, \ldots, n .
$$

Then the following linear system

$$
x^{\Delta}(t)=\operatorname{diag}\left(-c_{1}(t),-c_{2}(t), \ldots,-c_{n}(t)\right) x(t)
$$

admits an exponential dichotomy on $\mathbb{T}$, where $m\left(c_{i}\right)$ denote the mean-value of $c_{i}, i=$ $1,2, \ldots, n$.

Proof. According to Theorem 2.77 in [3], the linear system (5.7) has a unique solution

$$
x(t)=x_{0} e_{-c}\left(t, t_{0}\right)
$$

where $x\left(t_{0}\right)=x_{0},-c=\operatorname{diag}\left(-c_{1}(t),-c_{2}(t), \ldots,-c_{n}(t)\right)$. 
Now, we prove that $x(t)$ admits an exponential dichotomy on $\mathbb{T}$.

According to Theorem 3.2 and Theorem 3.5, one has

$$
m\left(c_{i}\right)=\lim _{T \rightarrow \infty} \frac{1}{T} \int_{t}^{t+T} c_{i}(s) \Delta s=\lim _{T \rightarrow \infty} \frac{1}{T} \int_{t_{0}}^{t_{0}+T} c_{i}(s) \Delta s>0, t_{0} \in \mathbb{T}, \quad i=1,2, \ldots, n .
$$

So there exists $T_{0}>0$, when $T>T_{0}$, one has

$$
\frac{1}{T} \int_{t_{0}}^{t_{0}+T} c_{i}(s) \Delta s>\frac{1}{2} m\left(c_{i}\right)=\frac{1}{T} \int_{t_{0}}^{t_{0}+T} \frac{1}{2} m\left(c_{i}\right) \Delta s, i=1,2, \ldots, n,
$$

that is

$$
\frac{1}{T} \int_{t_{0}}^{t_{0}+T}\left(c_{i}(s)-\frac{1}{2} m\left(c_{i}\right)\right) \Delta s>0, \quad i=1,2, \ldots, n,
$$

thus, for $T>T_{0}$, we have $c_{i}(t)>\frac{1}{2} m\left(c_{i}\right), i=1,2, \ldots, n$.

Case 1 . If $\mu(\eta)>0, \eta \in[s, t]_{\mathbb{T}}, s, t \in \mathbb{T}$, we have

$$
1-\frac{\mu(t) \frac{m\left(c_{i}\right)}{2}}{1+\mu(t) \frac{m\left(c_{i}\right)}{2}}>1-\mu(t) \frac{m\left(c_{i}\right)}{2}>1-\mu(t) c_{i}(t), \quad i=1,2, \ldots, n,
$$

then

$$
\int_{s}^{t} \frac{\log \left(1-\mu(\eta) c_{i}(\eta)\right)}{\mu(\eta)} \Delta \eta \leq \int_{s}^{t} \frac{\log \left(1-\frac{\mu(\eta) \frac{m\left(c_{i}\right)}{2}}{1+\mu(\eta) \frac{m\left(c_{i}\right)}{2}}\right)}{\mu(\eta)} \Delta \eta, \quad i=1,2, \ldots, n,
$$

thus

$$
\exp \left\{\int_{s}^{t} \frac{\log \left(1-\mu(\eta) c_{i}(\eta)\right)}{\mu(\eta)} \Delta \eta\right\} \leq \exp \left\{\int_{s}^{t} \frac{\log \left(1-\frac{\mu(\eta) \frac{m\left(c_{i}\right)}{2}}{1+\mu(\eta) \frac{m\left(c_{i}\right)}{2}}\right)}{\mu(\eta)} \Delta \eta\right\}, i=1,2, \ldots, n,
$$

that is,

$$
e_{-c_{i}}(t, s) \leq e_{\ominus} \frac{m\left(c_{i}\right)}{2}(t, s), \quad i=1,2, \ldots, n .
$$

Case 2. If $\mu(\eta)=0, \eta \in[s, t]_{\mathbb{T}}, s, t \in \mathbb{T}$, one cane easily obtain

$$
e_{-c_{i}}(t, s)=\exp \left\{\int_{s}^{t}-c_{i}(\eta) \Delta \eta\right\} \leq \exp \left\{\int_{s}^{t}-\frac{m\left(c_{i}\right)}{2} \Delta \eta\right\}=e_{\ominus \frac{m\left(c_{i}\right)}{2}}(t, s), \quad i=1,2, \ldots, n .
$$

Set $P=I$, we have

$$
\left|X(t) P X^{-1}(\sigma(s))\right|=\left|x_{0} e_{-c}\left(t, t_{0}\right) I x_{0}^{-1} e_{\ominus-c}\left(s, t_{0}\right)\right| \leq K e_{\ominus \frac{M}{2}}(t, s),
$$

where $K \geq 1, M=\min _{1 \leq i \leq n}\left\{m\left(c_{1}\right), m\left(c_{2}\right), \ldots, m\left(c_{n}\right)\right\}$. Therefore, $x(t)$ admits an exponential dichotomy with $P=I$ on $\mathbb{T}$. This completes the proof. $\square$ 
Example 5.1. Consider the following dynamic equation on an almost periodic time scale $\mathbb{T}=\bigcup_{i=1}^{\infty}[2 k, 2 k+1]$ :

$$
x^{\Delta}(t)=A x(t)+F(t)
$$

where

$$
A=\left(\begin{array}{cc}
-\frac{1}{16} & 0 \\
0 & -\frac{1}{16}
\end{array}\right), \quad F(t)=\left(\begin{array}{c}
\sin \sqrt{3} t+\frac{1}{t \sigma(t)} \\
\cos \sqrt{2} t+\frac{1}{t \sigma(t)}
\end{array}\right) \text { and } 0 \leq \mu(t)<16 .
$$

Obviously, $-A \in \mathcal{R}^{+}$. By Lemma 5.2, it is easy to know that the homogeneous equation of (5.8) admits an exponential dichotomy with $P=I$ on $\mathbb{T}$. Similar to Example 4.1, one easily to see that $F \in \tilde{\mathscr{P}} \mathscr{A} \mathscr{P}(\mathbb{T})_{2}$. By Theorem 5.2 and Theorem 2.77 in [3], one can obtain that (5.8) has a unique pseudo almost periodic solution:

$$
\begin{aligned}
x(t) & =\int_{-\infty}^{t} X(t) P X^{-1}(\sigma(s)) F(s) \Delta s-\int_{t}^{+\infty} X(t)(I-P) X^{-1}(\sigma(s)) F(s) \Delta s \\
& =\int_{-\infty}^{t} e_{-\frac{1}{16}}(t, \sigma(s))\left(\begin{array}{ll}
1 & 0 \\
0 & 1
\end{array}\right)\left(\begin{array}{c}
\sin \sqrt{3} s+\frac{1}{s \sigma(s)} \\
\cos \sqrt{2} s+\frac{1}{s \sigma(s)}
\end{array}\right) \Delta s .
\end{aligned}
$$

\section{Applications}

Application 1. Consider the following quasi-linear system

$$
x^{\Delta}=A(t) x+F+\mu_{0} G \circ(x \times \iota),
$$

where $\mu_{0} \in \mathbb{E}^{n} \backslash\{0\}, A(t)$ is a $n \times n$ almost periodic matrix, $F \in \tilde{\mathscr{P}} \mathscr{A} \mathscr{P}(\mathbb{T})_{n}$ and $G \in \tilde{\mathscr{P}} \mathscr{A} \mathscr{P}(\mathbb{T} \times D)_{n}$. We call the system

$$
x^{\Delta}=A(t) x+F
$$

the generating system of (6.1).

By Theorem 5.2, system (6.2) has a unique solution $x^{0} \in \tilde{\mathscr{P}} \mathscr{A} \mathscr{P}(\mathbb{T})_{n}$ if (5.2) admits an, exponential dichotomy. Now we have the following theorem about (6.1).

Theorem 6.1. If $F \in \tilde{\mathscr{P}} \mathscr{A} \mathscr{P}(\mathbb{T})_{n}, A(t)$ be almost periodic and (5.2) admits an exponential dichotomy. Let $x^{0} \in \tilde{\mathscr{P}} \mathscr{A} \mathscr{P}(\mathbb{T})_{n}$ be the unique solution of system (6.2) and denote $D=\left\{x \in \mathbb{E}^{n}:\left|x-x^{0}(t)\right| \leq a, t \in \mathbb{T}\right\}$, where $a>0$. Assume that

(i) $G \in \tilde{\mathscr{P}} \mathscr{A} \mathscr{P}(\mathbb{T} \times D)_{n}$ and $L>0$ such that

$$
\left|G\left(t, x^{\prime}\right)-G\left(t, x^{\prime \prime}\right)\right| \leq L\left|x^{\prime}-x^{\prime \prime}\right|, x^{\prime}, x^{\prime \prime} \in D, t \in \mathbb{T} ;
$$

(ii) $0<\left|\mu_{0}\right|<\min \left\{\frac{\alpha}{(2+\bar{\mu} \alpha) K L}, \frac{\alpha a}{(2+\bar{\mu} \alpha) K\|G\|}\right\}$, where $K$ and $\alpha$ are the same as those in Theorem 5.2, $\bar{\mu}=\sup _{t \in \mathbb{T}} \mu(t)$.

Then system (6.1) has a unique solution $x \in \tilde{\mathscr{P}} \mathscr{A} \mathscr{P}(\mathbb{T})_{n}$ such that $x \in D$ for all $t \in \mathbb{T}$. Furthermore, $\left\|x-x^{0}\right\| \rightarrow 0$ as $\mu_{0} \rightarrow 0$.

Proof. We construct a sequence of approximations by induction, starting with $x^{0}$ and taking $x^{k}$ to be the bounded solution of the system 


$$
\left(x^{k}\right)^{\Delta}=A(t) x^{k}+F+\mu_{0} G \circ\left(x^{k-1} \times \iota\right) .
$$

First, we show that $x^{k}$ exists, $x^{k} \in \tilde{\mathscr{P}} \mathscr{A} \mathscr{P}(\mathbb{T})_{n}$ and $x^{k}(\mathbb{T}) \subset D, k=0,1,2, \ldots$, where $x^{k}(\mathbb{T})$ denotes the value field of $x^{k}$. Obliviously, the conclusion holds for $k=0$ by the hypothesis. Assume that the conclusion holds for $k-1$. Then we shall show that the conclusion also holds for $k$. By Theorem 4.5, one can see that $G \circ\left(x^{k-1} \times \iota\right) \in \tilde{P} \mathscr{A} \mathscr{P}(\mathbb{T})_{n}$, so from Theorem 5.2, (6.4) has a unique solution $x^{k} \in \tilde{\mathscr{P}} \mathscr{A} \mathscr{P}(\mathbb{T})_{n}$. It follows from (6.2) and (6.4) that,

$$
\left(x^{k}-x^{0}\right)^{\Delta}=A(t)\left(x^{k}-x^{0}\right)+\mu_{0} G \circ\left(x^{k-1} \times \iota\right) .
$$

By (5.5), we have

$$
\left\|x^{k}-x^{0}\right\| \leq \frac{2+\bar{\mu} \alpha}{\alpha} K\left|\mu_{0}\right|\|G\| .
$$

Therefore, $x^{k}(\mathbb{T}) \subset D$, since

$$
\left|\mu_{0}\right| \leq \frac{\alpha a}{(2+\bar{\mu} \alpha) K\|G\|}
$$

Next, we show that $\left\{x^{k}\right\}$ is Cauchy sequence in $\tilde{\mathscr{P}} \mathscr{A} \mathscr{P}(\mathbb{T})_{n}$. Since

$$
\left(x^{k+1}-x^{k}\right)^{\Delta}=A(t)\left(x^{k+1}-x^{k}\right)+\mu_{0}\left[G \circ\left(x^{k} \times \iota\right)-G \circ\left(x^{k-1} \times \iota\right)\right],
$$

it follows from (5.5) and (6.3) that

$$
\begin{aligned}
\left\|x^{k+1}-x^{k}\right\| & \leq \frac{2+\bar{\mu} \alpha}{\alpha} K\left|\mu_{0}\right|\left\|G \circ\left(x^{k} \times \iota\right)-G \circ\left(x^{k-1} \times \iota\right)\right\| \\
& \leq \frac{2+\bar{\mu} \alpha}{\alpha} K\left|\mu_{0}\right| L\left\|x^{k}-x^{k-1}\right\| \\
& =\theta\left\|x^{k}-x^{k-1}\right\| \\
& \vdots \\
& \leq \theta^{k}\left\|x^{1}-x^{0}\right\|,
\end{aligned}
$$

where $0<\theta=\frac{2+\bar{\mu} \alpha}{\alpha} K\left|\mu_{0}\right| L<1$. This shows that $\left\{x^{k}\right\}$ is a Cauchy sequence in $\tilde{\mathscr{P}} \mathscr{A} \mathscr{P}(\mathbb{T})_{n}$. Since $\tilde{\mathscr{P}} \mathscr{A} \mathscr{P}(\mathbb{T})_{n}$ is a Banach space, there is an $x \in \tilde{\mathscr{P}} \mathscr{A} \mathscr{P}(\mathbb{T})_{n}$ such that $\left\|x^{k}-x\right\| \rightarrow 0$, when $k \rightarrow \infty$. It follows from (6.4) that $x$ is a solution of (6.1). It is clear that $\left\|x-x^{0}\right\| \rightarrow 0$, as $\mu_{0} \rightarrow 0$.

To show the uniqueness, let $x^{*}$ be another solution of (6.1). Similar to the discussion above, we have

$$
\left\|x-x^{*}\right\| \leq \theta\left\|x-x^{*}\right\|,
$$

this is a contradiction. The proof is complete. $\square$

Application 2. Let $D$ be a ball in $\mathbb{E}^{n}$ with center at origin and radius $r_{0}$. Consider the following system

$$
x^{\Delta}=A(t) x+G \circ(x \times \imath),
$$

where $A(t)$ is a $n \times n$ almost periodic matrix and $G \in \tilde{\mathscr{P}} \mathscr{A} \mathscr{P}(\mathbb{T} \times D)_{n}$. Set $\mathbb{B}=\left\{F \in \tilde{\mathscr{P}} \mathscr{A} \mathscr{P}(\mathbb{T} \times D)_{n}: F(\mathbb{T}) \subset D\right\}$, where $F(\mathbb{T})$ denotes the value field of $F$. 
$\mathbb{B}$ is a closed subset of $\tilde{P} \mathscr{A} \mathscr{P}(\mathbb{T} \times D)_{n}$. Therefore, $\mathcal{P}(\mathbb{T})$ is a complete metric space.

Theorem 6.2. Let $D, G, A(t)$, and $\mathcal{P}(\mathbb{T})$ be as those in the previous paragraph. Assume that, (5.2) admits an exponential dichotomy and the function $G$ satisfies,

$$
\frac{2+\bar{\mu} \alpha}{\alpha} K \sup _{(t, x) \in \mathbb{T} \times D}|G(t, x)| \leq r_{0} \text { where } \bar{\mu}=\sup _{t \in \mathbb{T}} \mu(t)
$$

and

$$
\left|G\left(t, x^{\prime}\right)-G\left(t, x^{\prime \prime}\right)\right| \leq L\left|x^{\prime}-x^{\prime \prime}\right|, x^{\prime}, x^{\prime \prime} \in D, t \in \mathbb{T}
$$

with $\frac{2+\bar{\mu} \alpha}{\alpha} K L<1$. Then (6.5) has a unique solution in $\tilde{\mathscr{P}} \mathscr{A} \mathscr{P}(\mathbb{T})_{n}$.

Proof. By Theorem 5.2, one can define the mapping $\tilde{T}: \mathbb{B} \rightarrow \tilde{\mathscr{P}} \mathscr{A} \mathscr{P}(\mathbb{T})_{n}$ by the fact that, for $F \in \mathbb{B}, \tilde{T} F$ is the unique pseudo almost periodic solution of the system

$$
x^{\Delta}=A(t) x+G \circ(F \times \imath) .
$$

We claim that $\tilde{T} B \subset B$ since by (5.5),

$$
\|\tilde{T} F\| \leq \frac{2+\bar{\mu} \alpha}{\alpha} K\|G \circ(F \times \iota)\| \leq r_{0} .
$$

The mapping is a contraction on $\mathcal{P}(\mathbb{T})$. In fact, for $F_{1}, F_{2} \in \mathbb{B}$, it follows from (5.5) and (6.6) that

$$
\begin{aligned}
\left\|\tilde{T} F_{1}-\tilde{T} F_{2}\right\| & \leq \frac{2+\bar{\mu} \alpha}{\alpha} K\left\|G \circ\left(F_{1} \times \iota\right)-G \circ\left(F_{2} \times \iota\right)\right\| \\
& \leq \frac{2+\bar{\mu} \alpha}{\alpha} K L\left\|F_{1}-F_{2}\right\| .
\end{aligned}
$$

Therefore, $\tilde{T}$ has a unique fixed point in $\mathcal{P}(\mathbb{T})$, which is the unique pseudo almost periodic solution of (6.5). The proof is complete. $\square$

\section{Acknowledgements}

This work is supported by the National Natural Sciences Foundation of People's Republic of China under Grant 10971183.

Authors' contributions

All authors contributed equally to the manuscript and typed, read and approved the final manuscript.

\section{Competing interests}

The authors declare that they have no competing interests.

Received: 27 October 2011 Accepted: 7 June 2012 Published: 7 June 2012

\section{References}

1. Agarwal, RP, Bohner, M, O'Regan, D, Peterson, A: Dynamic equations on time scales: a survey. Dynamic equations on time scales. J Comput Appl Math. 141, 1-26 (2002). doi:10.1016/S0377-0427(01)00432-0

2. Anderson, D, Bullock, J, Erbe, L, Peterson, A, Tran, H: Nabla dynamic equations on time scales. Panamer Math J. 13, 1-47 (2003)

3. Bohner, M, Peterson, A: Dynamic Equations on Time Scales. An Introduction with Applications. Birkhäuser, Boston (2001)

4. Laksmikantham, V, Sivasundaram, S, Kaymakcalan, B: Dynamical systems on measure chains. In Mathematics and its Applications, vol. 370,Kluwer, Dordrecht (1996)

5. Laksmikantham, V, Vatsala, AS: Hybrid systems on time scales. Dynamic equations on time scales. J Comput Appl Math. 141, 227-235 (2002). doi:10.1016/S0377-0427(01)00448-4

6. DaCunha, J: Stability for time varying linear dynamic systems on time scales. J Comput Appl Math. 176, 381-410 (2005). doi:10.1016/j.cam.2004.07.026

7. Zhou, JW, Li, YK: Sobolev's spaces on time scales and its applications to a class of second order Hamiltonian systems on time scales. Nonlinear Anal. 73, 1375-1388 (2010). doi:10.1016/j.na.2010.04.070

8. Li, YK, Zhang, TW: On the existence of solutions for impulsive duffing dynamic equations on time scales with Dirichlet boundary conditions. Abstr Appl Anal 2010, 27 (2010). Article ID 152460 
9. Li, YK, Chen, XR, Zhao, L: Stability and existence of periodic solutions to delayed Cohen-Grossberg BAM neural networks with impulses on time scales. Neurocomputing. 72, 1621-1630 (2009). doi:10.1016/j.neucom.2008.08.010

10. Liu, H, Xiang, X: A class of the first order impulsive dynamic equations on time scales. Nonlinear Anal. 69, 2803-2811 (2008). doi:10.1016/..na.2007.08.052

11. Li, YK, Shu, J: Multiple positive solutions for first-order impulsive integral boundary value problems on time scales. Bound Value Probl. 2011, 12 (2011). doi:10.1186/1687-2770-2011-12

12. Li, YK, Shu, J: Solvability of boundary value problems with Riemann-Stieltjes -integral conditions for second-order dynamic equations on time scales at resonance. Adv Differ Equ. 2011, 42 (2011). doi:10.1186/1687-1847-2011-42

13. Li, YK, Wang, C: Almost periodic solutions of shunting inhibitory cellular neural networks on time scales. Commun Nonlinear Sci Numer Simulat. 17, 3258-3266 (2012). doi:10.1016/j.cnsns.2011.11.034

14. Li, YK, Wang, C: Almost periodic functions on time scales and applications. Discr Dyn Nat Soc 2011, 20 (2011). Article ID 727068

15. Bochner, S: A new approach to almost periodicity. Proc Natl Acad Sci USA. 48, 2039-2043 (1962). doi:10.1073/ pnas.48.12.2039

16. Seifert, G: Almost periodic solutions and asymptotic stability. J Math Anal Appl. 21, 134-149 (1968)

17. Deysach, LG, Sell, GR: On the existence of almost periodic motion. Michgan Math J. 12, 87-95 (1965)

18. Seifert, G: On uniformly almost periodic sets of functions for almost periodic differential equations. Tohoku Math J. 34 301-309 (1982). doi:10.2748/tmj/1178229256

19. Miller, RK: Almost periodic differential equations as dynamical systems with applications to the existence of almost periodic solution. J Differ Equ. 1, 337-345 (1965). doi:10.1016/0022-0396(65)90012-4

20. Fink, AM: Almost periodic differential equations. In Lecture Notes in Mathematics, vol. 377,Springer, New York (1974)

21. Fink, AM, Seifert, G: Liapunov functions and almost periodic solutions for almost periodic systems. J Differ Equ. 5, 307-313 (1969). doi:10.1016/0022-0396(69)90045-X

22. David, C, Cristina, M: Invariant manifolds, global attractors and almost periodic solutions of nonautonomous deference equations. Nonlinear Anal. 56, 465-484 (2004). doi:10.1016/j.na.2003.09.009

23. Corduneanu, C: Almost Periodic Functions, 2nd edn.Chelsea, New York (1989)

24. N'Guérékata, GM: Almost Automorphic Functions and Almost Periodic Functions in Abstract Spaces. Kluwer, New York (2001)

25. Zhang, CY: Almost Periodic Type Functions and Ergodicity. Kluwer, New York (2003)

26. Zhang, CY: Pseudo almost periodic solutions of some differential equations. J Math Anal Appl. 151, 62-76 (1994)

27. Zhang, CY: Pseudo almost periodic solutions of some differential equations. II J Math Anal Appl. 192, 543-561 (1995)

28. Fink, A, Gatica, J: Positive almost periodic solutions of some delay epidemic equations. J Differ Equ. 83, 166-178 (1990). doi:10.1016/0022-0396(90)90073-X

29. Hino, Y, Minh, NV, Shin, JS: Almost Periodic Solutions of Differential Equations in Banach Spaces. Taylor and Francis, London (2002)

30. Caraballo, T, Cheban, D: Almost periodic and almost automorphic solutions of linear differential/difference equations without Favard's separation condition. I J Differ Equ. 246, 108-128 (2009). doi:10.1016/j.jde.2008.04.001

31. Boukli-Hacene, N, Ezzinbi, K: Weighted pseudo almost periodic solutions for some partial functional differential equations. Nonlinear Anal. 71, 3612-3621 (2009). doi:10.1016/j.na.2009.02.022

32. Dads, EHA, Cieutat, P, Ezzinbi, K: The existence of pseudo-almost periodic solutions for some nonlinear differential equations in Banach spaces. Nonlinear Anal. 69, 1325-1342 (2008). doi:10.1016/j.na.2007.06.037

33. Li, YK, Zhang, TW: Permanence and almost periodic sequence solution for a discrete delay logistic equation with feedback control. Nonlinear Anal Real World Appl. 12, 1850-1864 (2011). doi:10.1016/j.nonrwa.2010.12.001

34. Li, YK, Fan, XL: Existence and globally exponential stability of almost periodic solution for Cohen-Grossberg BAM neural networks with variable coefficients. Appl Math Modell. 33, 2114-2120 (2009). doi:10.1016/j.apm.2008.05.013

35. Li, YK, Wang, C: Uniformly almost periodic functions and almost periodic solutions to dynamic equations on time scales. Abstr Appl Anal 2011, 22 (2011). Article ID 341520

36. Cabada, A, Vivero, DR: Expression of the Lebesgue $\triangle$-integral on time scales as a usual Lebesgue integral; application to the calculus of $\Delta$-antiderivatives. Math Comput Modell. 43, 194-207 (2006). doi:10.1016/..mcm.2005.09.028

37. Zhang, J, Fan, $\mathrm{M}, \mathrm{Zhu}, \mathrm{H}$ : Existence and roughness of exponential dichotomies of linear dynamic equations on time scales. Comput Math Appl. 59, 2658-2675 (2010). doi:10.1016/j.camwa.2010.01.035

38. Bohner, M, Guseinov, GS: Double integral calculus of variations on time scales. Comput Math Appl. 54, $45-57$ (2007). doi:10.1016/j.camwa.2006.10.032

doi:10.1186/1687-1847-2012-77

Cite this article as: $\mathrm{Li}$ and Wang: Pseudo almost periodic functions and pseudo almost periodic solutions to dynamic equations on time scales. Advances in Difference Equations 2012 2012:77. 